\title{
A Collision-aware MAC Protocol for Efficient Performance in Wireless Sensor Networks
}

\author{
Hamid Hajaje $^{1}$ \\ Zine El Abidine Guennoun ${ }^{3}$ \\ Department of Mathematics \\ University Mohammed V \\ Rabat, Morocco
}

\author{
Mounib Khanafer ${ }^{2}$ \\ Department of Electrical and \\ Computer Engineering \\ College of Arts and Sciences \\ American University of Kuwait \\ Safat, Kuwait
}

\author{
Junaid Israr ${ }^{4}$ \\ Mouhcine Guennoun ${ }^{5}$ \\ Cisco Systems \\ Ottawa, Canada
}

\begin{abstract}
Both IEEE 802.11 and IEEE 802.15.4 standards adopt the CSMA-CA algorithm to manage contending nodes' access to the wireless medium. CSMA-CA utilizes the Binary Exponential Backoff (BEB) scheme to reduce the probability of packet collisions over the communication channel. However, BEB suffers from unfairness and degraded channel utilization, as it usually favors the last node that succeeded in capturing the medium to send its packets. Also, BEB updates the size of the contention window in a deterministic fashion, without taking into consideration the level of collisions over the channel. The latter factor has a direct impact on the channel utilization and therefore incorporating it in the computation of the contention window's size can have positive impacts on the overall performance of the backoff algorithm. In this paper, we propose a new adaptive backoff algorithm that overcomes the shortcomings of BEB and outperforms it in terms of channel utilization, power conservation, and reliability, while preserving the fairness among nodes. We model our algorithm using Markov chain and validate our system through extensive simulations. Our results show a promising performance for an efficient backoff algorithm.
\end{abstract}

Keywords-Wireless sensor networks; beacon-enabled IEEE 802.15.4; binary exponent backoff; adaptive backoff; fairness; power consumption; reliability; channel utilization

\section{INTRODUCTION}

The Binary Exponent Backoff (BEB) is an ingenious algorithm employed by both IEEE 802.11 and IEEE 802.15.4 standards to manage the wireless medium access among multiple competing nodes. BEB has been under the scope of extensive studies and have been shown to suffer from several performance pitfalls [1]-[5], [21]-[28], [65], [66] and [67]. Basically, [21], [22], and [23] provided detailed analysis of the MAC protocol, which implements BEB, in IEEE 802.11 to investigate its performance in terms of critical parameters, like throughput. The important conclusion drawn from these studies is that BEB suffers from a major shortcoming in terms of achieving high throughputs. It was proven that the practical performance, in terms of throughput, falls far behind the theoretical one and it is highly dependent on the number of nodes available. This observation motivated important research contributions that targeted enhancing BEB in IEEE 802.11-based networks (for example, see [53], [68], [69] and [70]). The same problem occurs in IEEE 802.15.4, which adopts a slightly modified version of BEB. IEEE 802.15.4's performance received a strong attention and many studies devised modified versions of BEB to achieve higher throughputs while preserving more power [6], [9-15], [18-19], [29]-[31], [35], [52], [54], [71], [72], [80]. In this paper we focus on the performance of BEB in IEEE 802.15.4-based wireless sensor networks (WSNs). We introduce changes into BEB that can overcome the limitations it experiences. The changes form a foundation for the new Adaptive Backoff Algorithm (ABA) that we model using Markov chain. We also conduct a simulation study to validate our proposed theoretical model. Our results are promising and pave the way for further improvements in future work. The rest of the paper is organized as follows. Section II overviews the BEB algorithm and highlights the problematic aspects of its functionality. In Section III we review the literature for contributions that targeted improving BEB in IEEE 802.15.4. In Section IV we describe ABA and model it mathematically using Markov chain. Section V describes the simulations we conducted to validate the developed mathematical model for $\mathrm{ABA}$ and compares the performance of ABA with a number of backoff algorithms proposed in the literature. Finally, Section VI concludes our work and envisions future research directions.

\section{OVERVIEW OF BEB IN IEEE 802.15.4 STANDARD}

The IEEE 802.15.4 standard defines the specifications of the PHY layer and the MAC sub-layer for low-rate personal area networks (LR-WPANs) [7], [8], [11], [13] and [32]. This standard suits the functionality of WSNs as it conforms to their distinguished requirements (like the need to preserve the resources of the sensor nodes [59]). The standard supports both star and peer-to-peer topologies. In the star topology, communications among nodes should go through a designated controller node called the PAN coordinator (or the coordinator for simplicity). In the peer-to-peer topology, however, direct communication between nodes is possible (and a coordinator still exists). The standard can operate in a beacon-enabled or a nonbeacon-enabled mode. The beaconenabled mode utilizes a superframe structure to control the communications over the wireless medium in a manner that reduces packet collisions. In Fig. 1, we depict the general structure of the superframe. As shown in the figure, the superframe is delimited by beacons that the coordinator sends periodically to synchronize the nodes. The superframe is constituted by active and inactive portions. The active portion 
consists of two main periods, namely, the contention access period (mandatory) and the contention free period (optional). The inactive portion, however, is used by the coordinator to conserve more power by conducting no activities. In this paper we ignore both the contention free period (CFP) and the inactive portion of the superframe.

During the CAP, nodes contend among themselves to secure an access to the wireless medium. The slotted CSMACA mechanism, that employs the BEB algorithm, is utilized here. In the rest of the paper, we focus on the beacon-enabled mode of IEEE 802.15.4.

The basic functionality of BEB is explained as follows. Before any transmission attempt, the backoff exponent $(B E)$ is initialized to macMinBE, a MAC attribute defined in the IEEE 802.15.4 standard with a default value of 3 . Then, the node backs off for a duration (i.e., contention window) chosen randomly from the range $\left[0,2^{B E}-1\right]$. Once the backoff period expires, the node proceeds for two clear channel assessments (CCAs). These assessments are needed to check whether the wireless medium is clear for commencing a transmission. Packet transmission starts only if the medium is found to be clear during the two CCAs. However, if either of the CCAs results in finding the medium busy, the value of $B E$ will be increased by one (up to a maximum of macMaxBE) and the node backs off again (the maximum number of allowed backoffs is macMaxCSMABackoffs). The idea behind incrementing $B E$ is to find its appropriate value that better adapts the duration of backoff to the level of activity over the communication medium. If $B E$ reaches its maximum, it cannot change unless successful/failed packet transmission occurs, or packet retransmission commences. In that case, $B E$ is reset to macMinBE. The packet will be dismissed if macMaxCSMABackoffs is crossed, and the CSMA-CA mechanism will start the BEB process over. Upon succeeding in transmitting a packet, an acknowledgement (ACK) packet is sent back by the receiver node. If the ACK packet is not received, the node attempts (up to a maximum of macMaxFrameRetries) to retransmit the packet. With every retry, the complete $\mathrm{BEB}$ procedure is re-applied. If macMaxFrameRetriesis crossed, the packet will be dismissed. It should be mentioned that basic time unit used by CSMACA is the aUnitBackoffPeriod, which we refer to it as time slot or time unit in the rest of the paper. Fig. 2 shows the CSMACA mechanism, including the BEB algorithm, with more details (the flow chart is taken from [32] with slight simplifications that do not affect the overall mechanism).

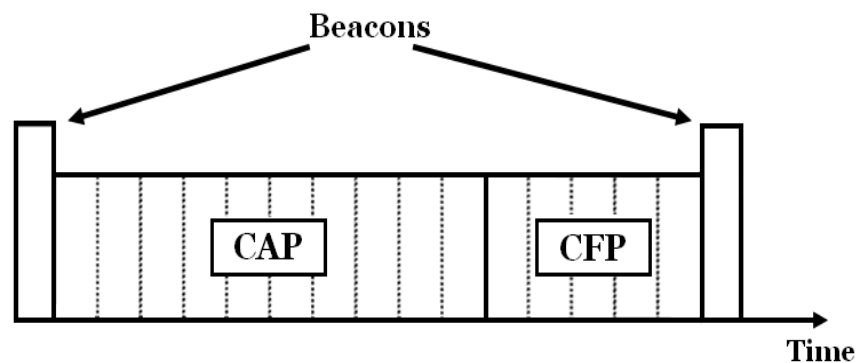

Fig. 1. Superframe Structure (Redrawn from [32]).

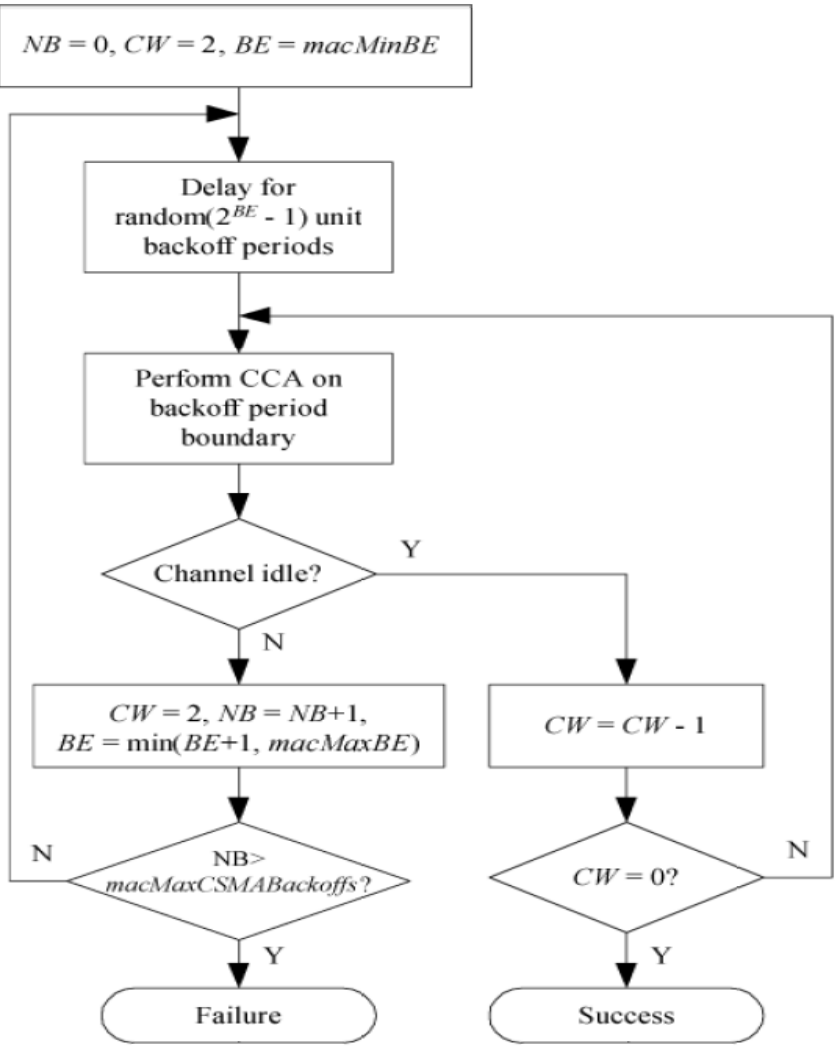

Fig. 2. CSMA-CA Mechanism [32]. NB Refers to the Number of Backoffs Done by the Node and CW is a Counter for the CCAs.

From the description of the BEB algorithm we can identify the reasons behind the degradation of the throughput in its performance evaluation. Clearly, BEB keeps on choosing the contention window randomly without taking into consideration the number nodes available in the network, the level of communication activity occurring on the medium, and the likelihood of packet collisions. A node increases $B E$ gradually, in steps of 1 , in attempts to transmit a packet and then resets $B E$ to its minimum, as explained before, without incorporating any information about its failed trials of transmissions or the intensity of the traffic in the medium. In other words, BEB is "memory-less" [24] as it keeps no information about the network status or conditions. On the other hand, BEB may have a problem of unfairness under saturation conditions [20][25]. This can be seen from noticing that a node that fails to access the medium tends to backoff for longer periods (because $B E$ keeps increasing as mentioned earlier), reducing its opportunity of sending its packets. However, a node that has just finished its successful transmission will reset its $B E$ to its minimum, which results in shorter backoff periods and thus higher chances of accessing the medium. That is, the last successful node is favored on the account of other nodes [26]. Clearly, under saturation conditions we will face a high rate of packet collisions, which leads to excessive power consumption and degraded throughput [33]. Furthermore, this behavior raises serious security concerns. A selfish node may deliberately tune its $B E$ such that it always achieves the minimum backoff period among the nodes. That way, the selfish node will access the 
medium much more frequently than the other nodes in the network (for more details on this misbehavior and how to mitigate it, see [55]-[58]). On the other hand, a node may act maliciously by tuning $B E$ to be at its maximum. This way, the node refrains from accessing the medium, which discourages other nodes from using this node as their next hop while forwarding packets. These functionality issues in BEB are far from being acceptable in WSNs. The next section focuses on the efforts proposed in the literature to modify BEB and enhance its performance in IEEE 802.15.4-based WSNs.

\section{RELATED WORK}

Research efforts proposed various methodologies to improve BEB. In the following we review some of these proposals.

Minooei and Nojumi studied improving BEB in the context of IEEE 802.11 [34]. Lee et al. investigated the performance of the algorithm in [34], which they call the NonOverlapping BEB (NO-BEB), in IEEE 802.15.4 networks [29]. NO-BEB modifies the way BEB selects the length of the contention window after an access failure. Basically, in order to reduce the level of contention over the medium, the contention window $(W)$ is randomly selected from the range $\left[W_{i-1}, W_{i}\right]$ rather than $\left[0, W_{i}\right]$, where $W_{i}$ is the contention window of the ith backoff stage [34]. This change guarantees that no overlapping with the previous range (that is, $\left[0, W_{i}\right]$ ) occurs. As a result, nodes experiencing different number of medium access failures have better chances of acquiring different contention windows from the non-overlapped regions. NO-BEB is modeled using Markov chain in [29] and shown to outperform BEB in terms of throughput, probability of collisions and average access delay. While NO-BEB introduces a creative methodology to incorporate the communication medium's status in the computation and selection of $W$, it still resets the latter to its minimum after each successful packet transmission. We mentioned in Section II that this behavior is problematic.

Woo et al. proposed the Knowledge-based Exponential Backoff (KEB) algorithm in [30]. The main target of KEB is to improve the throughput depending on the channel state information as collected by each node. Each node uses the Exponential Weighted Moving Average (EWMA), with a smoothing factor $\beta$, to compute locally the collision rate after each successful transmission. Based on that computation, the value of $B E$ is adjusted to achieve higher throughput. In other words, as the collision rate increases beyond a predefined collision threshold $\alpha, B E$ will be increased and thus nodes backoff for longer periods of time (in order to reduce the level of communications over the medium, which reduces the collisions). In contrast, as the collision rate remains below $\alpha$, nodes backoff for shorter periods of time, which improves the utilization of the communication channel. KEB has been modeled using Markov chain and then simulated to validate the analytical model. The provided results show that KEB outperforms BEB in terms of throughput. The main drawback of KEB is that its performance is mainly dependent on the value of $\alpha$. The authors provide a simulation study to find out the optimal values of these parameters that achieve the best throughput performance. However, this indicates that KEB cannot operate in an adaptive fashion and its performance will be governed by the targeted application.

Khan et al. introduce the Improved BEB (IBEB) algorithm in [31]. In IBEB each node, after specifying its $B E$, randomly selects an Interim Backoff (IB), which is restricted to be $10 \%$ to $40 \%$ of the specified backoff delay. The authors argue that this approach tends to reduce packet collisions since the probability of having two nodes randomly selecting the same $B E$ and $I B$ is quite low. The authors provided a simulation study to examine IBEB's performance in terms of latency, channel utilization, goodput, and average number of collisions. The results showed that IBEB outperforms BEB in terms of these parameters. We highlight that IBEB may suffer from major degradation as the number of nodes increases in the network. This is because the pool of IBs is so narrow that many nodes will happen to select the same IB, which contributes to higher packet rates of collisions.

Zhu et al. in [39] modify the CSMA-CA algorithm and propose the Linear Increase Backoff (LIB) scheme to enhance the performance in term of packet delay. The idea of LIB is that the backoff counter, upon sensing any of the two CCAs busy, increases linearly instead of exponentially (which is the case in BEB). The authors analyze the behavior of LIB using a comprehensive Markov model. Using this model, they extract formulas to describe the packet delay, energy consumption and throughput of unsaturated, unacknowledged traffic. Simulations of LIB show that it achieves a superior performance in terms of delay, throughput, and energy conservation. LIB is mainly designed for time-critical monitoring and detection applications, and therefore, minimization of the delay is the main target of this scheme.

In [73], the authors have proposed a backoff algorithm, named Waiting Backoff Algorithm (WBA), in order to enhance both the delay and the throughput performance of IEEE 802.11 MAC protocol. The main idea behind this algorithm is to observe the waiting time of each station during the backoff stage and then estimate the size of the contention window in the network. The authors have conducted some simulation experiences to show WBA enhances the basic backoff algorithm for some specific number of stations.

J. Sartthong and S. Sittichivapak have employed the mathematical optimization function theory to propose another backoff algorithm called Contending Stations Backoff Algorithm (CSBA) [74]. This paper includes a comparison results with $\mathrm{BEB}$ and other backoff algorithms and shows some improvements in term of saturation throughput efficiency. The same authors have extended this work to achieve more performance by this time by introducing [75] both a Binary Exponential Increment Half Decrement backoff algorithm and a discrete Markov chain model called the Fixed Backoff stages and Fixed Contention windows, named BEIHD and FBFC respectively.

In [68], Q. Liu and A. Czylwik have tackled the collision problems in WSN by proposing a collision-aware backoff mechanism (CABEB). Their main idea consists of involving a collision aware module that captures the channel state and adapts the MAC layer based on the collision probability to control the length of backoff period. 
In [76], R. TejaChekka et al. have proposed an Adaptive Binary Exponential Backoff algorithm to handle multiple channel access issue by avoiding the collision at the sender side and the buffer overflow at receiver side without degrading channel utilization and throughput efficiency.

In [69], M. Shurman et al. have analyzed the three backoff algorithms BEB, I-BEB and E-BEB and highlighted their limitations. Then, they proposed the New Binary Exponential Backoff (N-BEB) algorithm to improve channel access fairness while preserving the channel throughput. It consists of improving contention window configuration, based on the number of successful and unsuccessful transmissions.

In [77], the authors have tackled the BEB problems by providing two main contributions. First, they proposed an adaptive backoff mechanism through which nodes can dynamically adjust the backoff period according to the actual status of the network. Second, they proposed a priority-based service-differentiation mechanism to provide multi-levels differentiated services in order to meet different QoS requirements.

In [78], the authors have tackled the 802.15.4 performance issues by proposing a backoff mechanism in which the backoff duration is adaptively chosen, when WiFi transmissions are detected during the clear channel assessment. They also consider erroneous decisions regarding the type of packet detected during the CCA and prove that the proposed algorithm remains efficient. Some simulation experiences have been conducted with 10 nodes to demonstrate the feasibility of such technique.

In [70], X. Liu et al. have addressed BEB drawbacks by using a different approach. They proposed a backoff algorithm, which adopts a retransmission counter to measure the network congestion situation. The proposed algorithm also divides the contention window interval into small intervals, and in different intervals, it leverages different backoff strategies. They measured the performance of this approach that showed slight improvements in throughput rate for some specific cases.

In [79], Y. Huang et al. have proposed a Synchronized Contention Window-based backoff algorithm, named SCW. In SCW algorithm, each station actively tracks the transmissions and when the channel state is changed, resetting the CW value synchronizes the CW of each station, which participates in the competition. This way, it makes each station get the medium access grant with the same probability in next channel contention. The experimental results of this technique show some improvement in the case o large number of stations.

\section{ADAPTIVE BACKOFF ALGORITHM}

Based on the discussions outlined in Sections II and III, we now introduce the new Adaptive Backoff Algorithm (ABA). The pivotal objective behind ABA is to improve the channel utilization $(U)$ in the WSN. ABA achieves higher levels of channel utilization than is possible with BEB while keeps the power consumption at the lowest possible level (which is a primary requirement to prolong the lifetime of the WSN). Preliminary to designing/modifying any backoff algorithm, it is essential to understand the factors that play the role of degrading $U$. From one side, whenever the nodes select long backoff periods, the wireless medium is forcibly kept idle for a long duration of time and thus $U$ is affected. From another side, as the rate of packet collisions rises, the useful communication activities over the medium are affected and $U$ is reduced as a result. Failing to consider these two factors results in partially effective backoff algorithms.

The problem with BEB is that it provides a deterministic solution that keeps on updating the length of the contention window based on predefined steps. This is the main shortcoming of BEB. We need a probabilistic solution that can involve the status over the wireless medium in the computation of the contention window. Based on that, ABA proposes that the probability of collision $\left(P_{c}\right)$ be used in updating the value of the contention window. This approach guarantees that the contention window will be adapted to conditions over the communication channel. Stated differently, the value of the contention window will be updated as follows:

$W(t)=P_{c}(t) W_{\max }$

where, $W(t)$ is the selected contention window at time $t$ ( $W(t)$ and $W$ will be used interchangeably in the rest of the paper), $W_{\text {max }}$ is IEEE 802.15.4's maximum contention window (set to $2^{\text {macMaxBE}}$ ), and $P_{c}(t)$ is the probability of collisions at time $t$ (In the rest of the paper, $P_{c}(t)$ and $P_{c}$ will be used interchangeably). $P_{c}$ is computed locally at each node by knowing the proportion of packets that suffered from collisions. This proportion is computed as $n_{c} /\left(n_{s}+n_{c}\right)$, where $n_{c}$ and $n_{s}$ are, as observed by any node, the total number of collided packets and the total number of successfully transmitted packets, respectively (note that in case of unacknowledged traffic, we assume that a mechanism at higher layers is available to advise the MAC of a collision after a certain timeout). According to (1), upon having a packet to send, the node backs off for a duration that cannot exceed $W_{\text {max }}$. In case that CCA1 or CCA2 reveal that the medium is busy, the backoff process is repeated macMaxCSMABackoffs times before discarding the packet. On the other hand, upon experiencing an idle medium after the two CCAs, the packet is sent. In case of a packet collision, the node updates its $W(t)$ based on the number of collisions it faces and resends the packet. If the packet continues to collide more than macMaxFrameRetries times, the packet will be discarded. Equation (1) indicates that, as the rate of collisions increases (decreases), the node utilizes an extended (a shortened) backoff period. This is anticipated to significantly reduce (increase) the contention among nodes, and also allows them better chances of successful transmission.

The overall result is enhanced channel utilization in the network. ABA requires no hardware upgrades and requires simplified computations that requires low power consumption (as we demonstrate in Section V) and can be easily implemented in sensor nodes' platform. In Fig. 3 we show the flow diagram of ABA.

We now develop the mathematical model for ABA based on Markov chain (see [41]-[51] for extensive studies on how to model IEEE 802.15.4's BEB using Markov chain). The 
model covers saturated traffic under both acknowledged and unacknowledged traffic conditions. We should mention that saturation conditions are typical in important research areas, like Wireless Body Area Networks (WBAN) (see [63] and [64] for examples).

In Fig. 4 we illustrate a two-dimensional Markov chain that covers all the states a node goes through to access the medium, using ABA, while working under saturated traffic conditions. The latter means that the node has always a packet to send. Each state in our Markov model is distinguished by a pair $(i, j)$, where $i$ can be $0,-1$, or -2 , to refer to the backoff/CCA states, successful transmission states, or collision states, respectively. The $j$ index will be clarified in the following. States $(0, j)$, where $j \in[1, W-1]$, refer to the duration of backoff during which the node is involved in no activity, waiting for its backoff counter to expire. States $(0,0)$ and $(0,-1)$ correspond to CCA1 and CCA2, respectively. States $(-1, j)$, where $j \in\left[0, L_{s}-1\right]$, correspond to the duration spent to successfully transmit a packet. Finally, States $(-2, j)$, where $j \in\left[0, L_{c}-1\right]$, correspond to the time wasted due to a packet collision. The probability of finding the medium busy during CCA1 (CCA2) is denoted as $\alpha(\beta)$ (an explanation on the difference between $\alpha$ and $\beta$ is detailed in [38]).

The state transition probabilities of our Markov chain are as follows:

$$
\begin{aligned}
& P(0, j-1 \mid 0, j)=1 \text { for } 0<j \leq W-1 \\
& P(0, j \mid 0,0)=\frac{W-j}{W} \quad \text { for } j \geq 0 \\
& P(0,-1 \mid 0,0)=1-\alpha \\
& P(-1, j \mid 0,0)=(1-\alpha)(1-\beta)\left(1-P_{c}\right) \text { for } 0 \leq j \leq L_{s}- \\
& 1
\end{aligned}
$$

$$
P(-2, j \mid 0,0)=(1-\alpha)(1-\beta) P_{c} \quad \text { for } 0 \leq j \leq L_{c}-
$$

1

$P(0, j \mid-1, j$ or $-2, j)=\frac{1}{W}$ for $j \geq 0$

Equation (2) captures how the backoff counter decrements before attempting any packet transmission. Equation (3) describes the probability of backing off given that the medium was found busy during CCA1 or CCA2. Note that $W$ in this equation will be updated according to (1). It is important to point out that deriving (3) is attained by summing all the transition probabilities starting from state $(0,0)$ and ending at any of the states $(0,0), \ldots,(0, W-1)$. The summation includes the probabilities: $(\alpha+(1-\alpha) \beta) / W$ (to transit from state $(0,0)$ to any of the backoff states $(0, j)$, where $j \in[0, W-1])$, $(1-\alpha)(1-\beta)\left(1-P_{c}\right) / W$ (to transit from state $(0,0)$ to any of the backoff states, after a successful transmission), (1 $\alpha)(1-\beta) P_{c}$ (to transit from state $(0,0)$ to any of the backoff states, after a packet collision), and the probability of being at the state preceding the selected backoff state (so, if backoff state $(0,1)$ was randomly selected, we should include the probability of being at backoff state $(0,2)$ in our summation, and so on). Equation (4) states the probability of initiating CCA2 given that CCA1 was successful. Equation (5) is the probability of successfully sending a packet after two successful CCAs while Equation (6) is the probability of having a packet collision after those CCAs. Finally, Equation (7) describes the even probability of choosing a contention window after packet transmission/collision. Assuming that $s(t)$ and $c(t)$ are the stochastic processes representing the backoff stage and the state of the backoff counter, respectively, we now write the stationary distribution of our Markov chain to be $b_{i, j}=\lim _{t \rightarrow \infty} P(s(t)=i, c(t)=j)$,

where $i \epsilon[-2,0]$ and $j \in\left[-1, \max \left(W-1, L_{s}-1, L_{c}-\right.\right.$ $1)$ ]. We now derive the closed form expressions for this distribution.

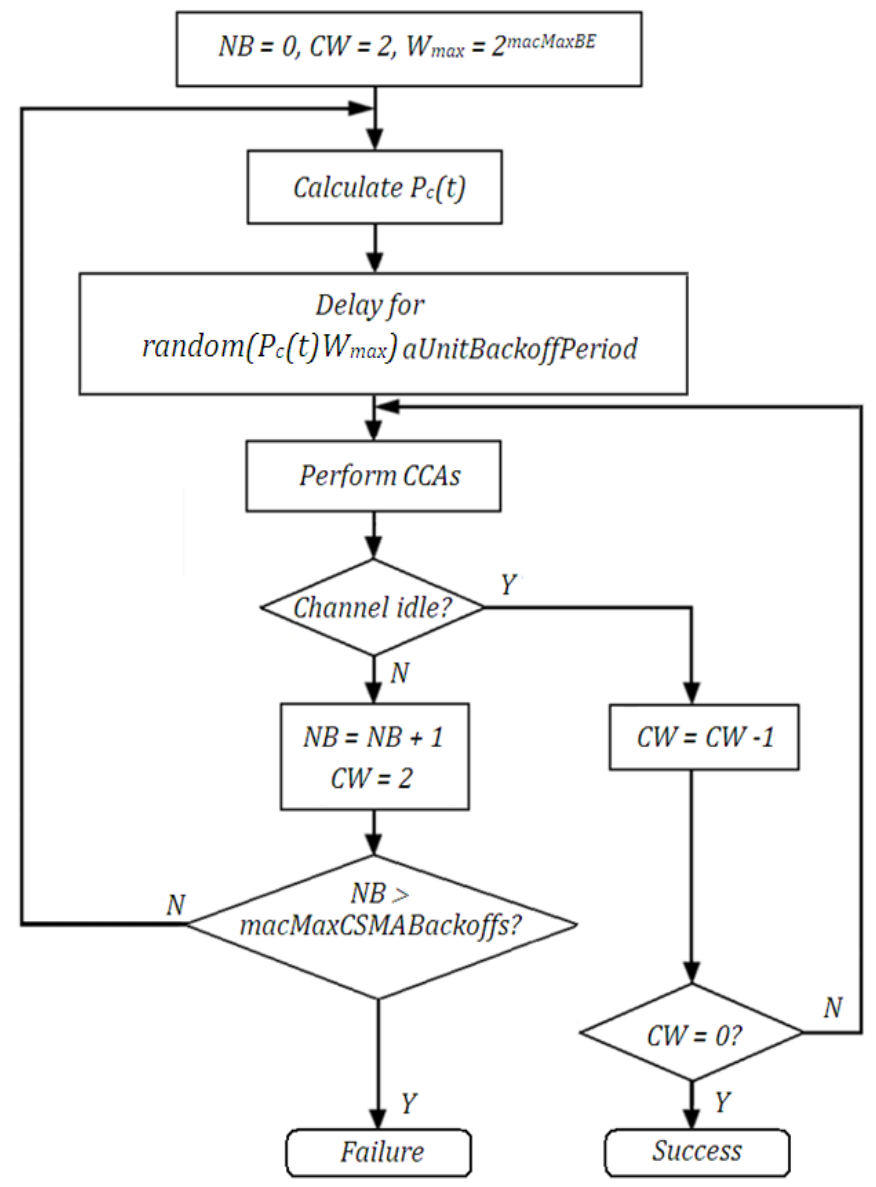

Fig. 3. ABA Algorithm.

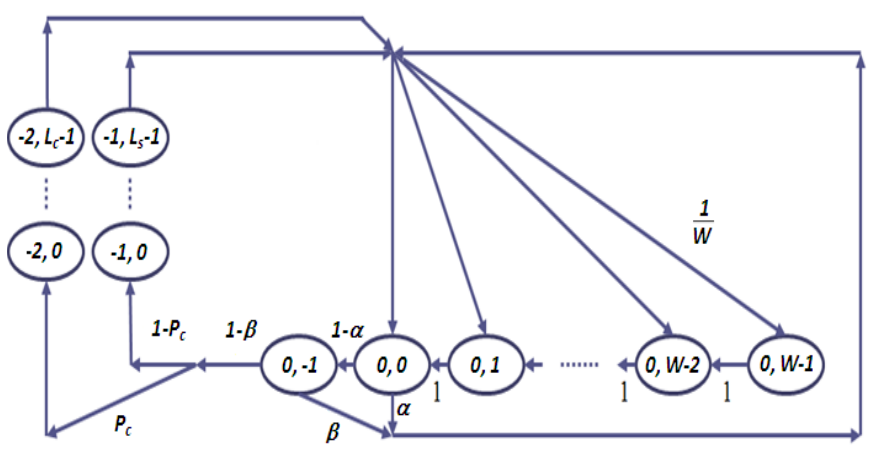

Fig. 4. Markov Chain of ABA Algorithm Under Saturated Traffic Conditions. 
By the normalization condition, we have the following formula:

$\sum_{j=1}^{W-1} b_{0, j}+\sum_{j=0}^{L_{S}-1} b_{-1, j}+\sum_{j=0}^{L_{c}-1} b_{-2, j}+b_{0,0}+b_{0,-1}=1$

The first term in (8) refers to the backoff states, the second term refers to the packet transmission states, the third term refers to the packet collision states, and the fourth and the fifth terms refer to the CCA1 and CCA2 states, respectively. We depend on the Equations (2)-(7) to find the mathematical expression for each of these terms. The latter will be expressed in terms of $b_{0,0}$, for which a closed form expression will be derived later. Based on (2), (3), and (7) we can write:

$\sum_{j=1}^{W-1} b_{0, j}=\sum_{j=1}^{W-1} \frac{W-j}{W} b_{0,0}=\frac{W-1}{2} b_{0,0}$

Next, based on (5) and (6) we have:

$\sum_{j=0}^{L_{S}-1} b_{-1, j}=L_{s}(1-\alpha)(1-\beta)\left(1-P_{c}\right) b_{0,0}$

$\sum_{j=0}^{L_{c}^{-1}} b_{-2, j}=L_{c}(1-\alpha)(1-\beta) P_{c} b_{0,0}$

Finally, based on (4) we obtain the following formula:

$b_{0,-1}=(1-\alpha) b_{0,0}$

The probability of collision, $P_{c}$, is formulated in [16] and [17] as follows:

$P_{c}=1-(1-\tau)^{N-1}$

Equation (13) is formulated based on the observation that a collision will not happen if, out of $N$ nodes, only one node is at CCA1, while the remaining $N$-1 nodes are at any state other than CCA1. Assuming that the probability that a node initiates CCA1 is $\tau$, the probability of having no collisions in the network will be $(1-\tau)^{N-1}$. Therefore, $P_{c}$ will be the complement of the latter term, which gives Equation (13). We point out, however, that when a certain node is initiating its CCA1, it is not quite accurate to assume that the remaining $N$ 1 nodes can be at any state other than CCA1. These nodes can only be in the backoff states. They cannot be, for example, in the state $(-2,0)$. Otherwise, the original node, at the CCA1 state, cannot send out its packet in the first place. Therefore, we accept that Equation (13) provides a reasonable approximation of $P_{c}$ in the network, but we expect that it may cause some deviations from the actual behaviour, as we demonstrate later in Section V.

The abovementioned definition of $\tau$ indicates that it is equal to $b_{0,0}$. Therefore, we can now substitute Equations (9)(13) into (8) and solve for $\tau$. The substitution results in the following formula:

$\tau=\frac{2}{3-2 \alpha+2(1-\alpha)(1-\beta)\left[L_{S}+P_{c}\left(L_{c}-L_{S}\right)\right]+W}$

Both $L_{s}$ and $L_{c}$ are defined in the IEEE 802.15.4 standard (see [32]). $W$ and $P_{c}$ has already been defined in (1) and (13), respectively. Therefore, we need to find mathematical expressions for $\alpha$ and $\beta$ to solve for $\tau$. These expressions strongly depend on whether the communicated traffic is acknowledged or not. Pollin et al. have provided in [37] (also, see [39]) a detailed study in this direction and we adopt their findings in this paper. In case of unacknowledged traffic, we have the following expressions:

$\alpha=L\left(1-(1-\tau)^{N-1}\right)(1-\alpha)(1-\beta)$

$\beta=\frac{1-(1-\tau)^{N-1}}{2-(1-\tau)^{N-1}}$

where, $L$ in (15) refers to the length of the packet to be sent. On the other hand, for acknowledged traffic we have the following expressions:

$\alpha=P_{c}(1-\alpha)(1-\beta)\left(L+L_{a c k} \frac{N \tau(1-\tau)^{N-1}}{1-(1-\tau)^{N}}\right)$

$\beta=\frac{1-(1-\tau)^{N-1}+N \tau(1-\tau)^{N-1}}{2-(1-\tau)^{N}+N \tau(1-\tau)^{N-1}}$

where, $L_{\text {ack }}$ in (17) refers to the length of the ACK packet. We should mention that Equations (16) and (18) are approximated for large $N$ (see [37] and [39] for details).

Equation (14) along with Equations (15)-(16) (or Equations (17)-(18)) for unacknowledged (or acknowledged) traffic form a nonlinear equation system of three variables, namely, $\tau, \alpha$, and $\beta$. This system of equations can be solved using numerical methods to find the operating point of the network.

It is worth mentioning that the Markov model depicted in Fig. 4 is much simpler than the one provided in [36], [37], [38], and [39]. In these studies, the authors show all the backoff and packet transmission retries stages to study the functionality of the MAC layer in IEEE 802.15.4. However, although our model shows these stages augmented as one stage, yet, as we show later, we are able to capture the main characteristics of the MAC layer and derive all the formulas that describe its functionality. We will see in the next section that showing the backoff and retries stages is just needed for the sake of computing the reliability of the system. We will follow a methodology that helps in deriving a mathematical formula for the reliability without undermining the validity of the model in Fig. 4.

\section{A. Channel Utilization Under ABA}

The Channel Utilization $(U)$ parameter measures how efficiently we are utilizing the wireless medium to successfully transmit packets. In an unacknowledged traffic situation, $U$ refers to the probability that a node sends a packet successfully. However, in an acknowledged traffic situation, the node should receive back the ACK packet in order to consider the transmission successful. By examining the model in Fig. 4, we notice that channel utilization is defined as follows:

$U=N L \tau(1-\alpha)(1-\beta)\left(1-P_{c}\right)$

which reduces to:

$U=N L \tau(1-\alpha)(1-\beta)(1-\tau)^{N-1}$

where, $N$ is included in the computation in order to find the total $U$ achieved from the successful transmissions of all the nodes in the network. 


\section{B. Power Consumption Under ABA}

It is essential to study the performance of ABA in terms of power consumption. This is because sensor nodes are batterypowered and any proposed algorithm for WSNs should not deplete the nodes' power resources at a high pace.

Under ABA, a node can be in any of the following states: backoff states, CCA states, packet transmission (with either success or collision) states. The power consumed at a node, denoted $E_{\text {total }}$, is the total summation of the power consumed at each of these states:

$E_{\text {total }}=E_{\text {idle }}+E_{C C A}+E_{t x}+E_{r x}$

$E_{\text {idle }}$ is the total power consumed during the backoff states:

$E_{\text {idle }}=P_{\text {idle }} \sum_{j=1}^{W-1} b_{0, j}=P_{\text {idle }} \frac{W-1}{2} b_{0,0}$

$E_{C C A}$ is the total power consumed during the two CCA states:

$E_{C C A}=P_{C C A}\left(b_{0,0}+b_{0,-1}\right)=P_{C C A}(2-\alpha) b_{0,0}$

where, $P_{\text {idle }}$ and $P_{C C A}$ to refer to the average power consumed during a backoff state and a CCA state, respectively.

We should pay a careful attention to $E_{t x}$, the total power consumed during packet transmission. The value of $E_{t x}$ depends on the type of traffic assumed, whether it is acknowledged on not. According to IEEE 802.15.4 [32], if the traffic is acknowledged, the node, after sending a packet (thus, $P_{t x}$ is considered), becomes idle for a period of one time slot (thus, $P_{\text {idle }}$ is considered) before it starts sensing the ACK packet. If the ACK packet is sensed, we should consider the average power consumed during reception $\left(P_{r X}\right)$. If the ACK packet is not sensed after a period of $L_{a c k}$, or in case of having a collision, the node becomes idle for an extra time slot (thus, $P_{\text {idle }}$ is considered) before proceeding to sending the next packet. Therefore, as already noted in [36], to compute the total power consumed while sending acknowledged traffic, we have the following formula:

$$
\begin{aligned}
E_{t x}= & P_{t x}\left(\sum_{j=0}^{L_{s}-1} b_{-1, j}+\sum_{j=0}^{L_{c}-1} b_{-2, j}\right)+P_{i d l e}\left(b_{-1, L_{s}}+b_{-2, L_{c}}\right) \\
& +P_{r x} \sum_{j=L_{s}+1}^{L_{s}+L_{a c k}} b_{-1, j}+P_{\text {idle }} \sum_{j=L_{c}+1}^{L_{c}+L_{a c k}+1} b_{-2, j}
\end{aligned}
$$

The first term in Equation (23) considers the transmission of the packet, whether it is successful or not. The second term corresponds to the additional time slot in waiting for the ACK packet. The third term evaluates the average power consumed while receiving the ACK packet. Therefore, the summation starts at $L_{s}+1$ which takes into consideration that we wait for $L_{s}$ time slots and then one extra time slot before receiving the ACK. Finally, the fourth term corresponds to the time slot waited in the cases of having a collision or losing the ACK packet.

In case of unacknowledged traffic, only the first term of Equation (23) is considered.
Finally, $E_{r x}$, the average power consumed during reception of packets, for both acknowledged and unacknowledged traffics, is expressed as follows:

$E_{r x}=P_{r x} \sum_{j=0}^{L_{s}-1} b_{-1, j}$

\section{Reliability Under $A B A$}

Reliability $(R)$ is defined in [36] as the probability of achieving a successful packet reception. In other words, $R$ provides us a measure of how efficient ABA is in improving the possibility of transferring a packet to its destination. Under ABA, a packet is dismissed if we exceed either macMaxCSMABackoffs or macMaxFrameRetries. That is, a node goes through multiple backoff stages, in case of busy CCAs, and/or multiple transmission retries, in case of repeated collisions, before dismissing a packet. Therefore, formulating $R$ depends on finding the probability of avoiding the dismissal of a packet. As the likelihood of dismissing a packet diminishes, it means that the system is more reliable. Therefore, the reliability is defined as follows:

$R=\frac{\pi_{s}}{\pi_{s}+\pi_{f}}$

Where, $\pi_{s}$ is the probability of having successful transmissions and $\pi_{f}$ is the probability of having failed transmissions. Note that failed transmissions include both collided packets and discarded packets. While $\pi_{s}$ is known from Equation (5), special attention is needed to formulate $\pi_{f}$. We develop the finite-state machines (FSMs) shown in Fig. 5 and use them to accomplish that. The FSM in Fig. 5(a) shows that a node, as it goes from state $(0,0)$ and ends back at it, may encounter a successful transmission (S), a packet collision (C), or a busy channel (B). Fig. 5(a), however, does not show the multiple bakoff stages and packet transmission retries a node may experience while attempting to send a packet. In other words, the $B$ and $C$ states are in fact constituted by multiple stages. These stages are shown in Fig. 5(b) and 5(c). Note that these two FSMs can be merged to show the complete system, but we avoid that to simplify our derivations. Based on Equations (3)-(6), we can directly see that $x=\alpha+(1-\alpha) \beta, y=(1-\alpha)(1-\beta) P_{c}$, and $z=$ $(1-\alpha)(1-\beta)\left(1-P_{c}\right)$. These equations can be also inferred by noticing the transitions in Fig. 5(a). The FSM in this figure is interpreted as follows. As a node finds the channel busy, it has a probability of $x$ to find the channel busy again. On the other hand, it may succeed to send its packet or face a packet collision with probabilities $z$ and $y$, respectively. After succeeding in sending a packet, a node may be successful in sending the next packet with a probability of $z$. Otherwise, the node may find the channel busy with probability $x$ or suffer from a collision with probability $y$. Finally, as the node experiences a collision, it may encounter another collision with probability $y$, succeed in sending the packet with probability $z$, or find the channel busy with probability $x$.

In Fig. 5(b) and 5(c) we capture the fact that, during a single cycle, a node may go through macMaxCSMABackoffs (denote as $m$ in Fig. 5(b)) backoff stages and macMaxFrameRetries (denote as $n$ in Fig. 5(c)) collisions before discarding a packet (note that $\pi_{C_{i}}$ denote the probabilities to suffer from a collision after finding the 
channel busy for $i$ times). Therefore, in order to find $R$, we need to find the probability that the system backs off for $m+1$ times or experiences a collision for $n+1$ time. If we assume $A$ to be a random variable that denotes the number of backoff stages the node has gone through, and $B$ to be a random variable that denotes the number of collisions occurred, then Equation (25) can be rewritten as follows:

$R=\frac{\pi_{S}}{\pi_{S}+P(A=m+1)+P(B=n+1)}$

$P(A=m+1)$ and $P(B=n+1)$ can be found using the FSMs in Fig. 5(b) and 5(c), respectively. Towards that end, if we have the transition matrices $P_{1}$ (for Fig. 5(b)) and $P_{1}$ (for Fig. 5(c)), then there exist the stationary distributions $\pi_{1}$ and $\pi_{2}$, such that $P_{1} \times \pi_{1}=\pi_{1}$ and $P_{2} \times \pi_{2}=\pi_{2}$. The latter relationships are expanded, respectively, as follows:

$$
\begin{aligned}
& \begin{array}{lllllll}
S & B_{1} & B_{2} & \cdots & B_{m} & B_{m+1} & C
\end{array} \\
& \begin{array}{c}
S \\
B_{1} \\
B_{2} \\
\vdots \\
B_{m} \\
B_{m+1} \\
C
\end{array} \quad\left[\begin{array}{ccccccc}
Z & z & Z & \cdots & Z & z & Z \\
x & 0 & 0 & \cdots & 0 & x & x \\
0 & x & 0 & \cdots & 0 & 0 & 0 \\
\vdots & \vdots & \ddots & \ddots & \vdots & \vdots & \vdots \\
0 & 0 & 0 & \ddots & 0 & 0 & 0 \\
0 & 0 & 0 & \cdots & x & 0 & 0 \\
y & y & y & \cdots & y & y & y
\end{array}\right] \times\left[\begin{array}{c}
\pi_{S} \\
\pi_{B_{1}} \\
\pi_{B_{2}} \\
\vdots \\
\pi_{B_{m}} \\
\pi_{B_{m+1}} \\
\pi_{C}
\end{array}\right]=\left[\begin{array}{c}
\pi_{S} \\
\pi_{B_{1}} \\
\pi_{B_{2}} \\
\vdots \\
\pi_{B_{m}} \\
\pi_{B_{m+1}} \\
\pi_{C}
\end{array}\right] \\
& \begin{array}{llllllll}
S & C_{1} & C_{2} & \cdots & C_{m} & C_{m+1} & B
\end{array}
\end{aligned}
$$

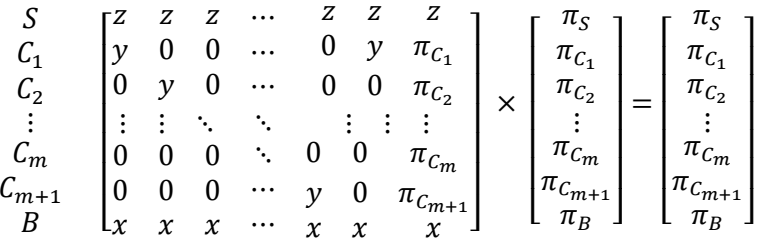

In (27) the stationary distribution $\pi_{1} i \mathrm{~s}$ defined as $\left[\begin{array}{lllllll}\pi_{S} & \pi_{B_{1}} & \pi_{B_{2}} & \ldots & \pi_{B_{m-1}} & \pi_{B_{m}} & \pi_{C}\end{array}\right]$, where $\pi_{B_{k}}$ is the probability of being in the $k$ th backoff stage. On the other hand, $\pi_{2}$ is defined as $\left[\begin{array}{lllllll}\pi_{S} & \pi_{C_{1}} & \pi_{C_{2}} & \ldots & \pi_{C_{n-1}} & \pi_{C_{n}} & \pi_{B}\end{array}\right]$ for (28), where $\pi_{C_{r}}$ is the probability of experiencing the $r$ th collision.

Based on (27) and (28), we can write the following formulas (see Appendix A for the detailed derivations):

$P(A=m+1)=\pi_{B_{m+1}}=\frac{x^{m+1}(1-x)}{1-x^{m+1}}$

$P(B=n+1)=\pi_{C_{n+1}}=\frac{(1-x-y) y^{n+1}}{(1-x)^{n+1}-y^{n+1}}$

Finally, with the knowledge of Equations (26), (28), and (29), we can now formulate the reliability as follows:

$R=\frac{1}{1+\frac{(1-x) x^{m+1}}{\left(1-x^{m+1}\right)(1-x-y)}+\frac{y^{n+1}}{(1-x)^{n+1}-y^{n+1}}}$

\section{Channel Collision Time Under ABA}

An efficient backoff algorithm should prove effectiveness in reducing the rate of collisions in the wireless medium. This is essential because not only it improves the utilization of the communication channel, but also reduces the consumption of power due to useless activities.

We aim in this subsection at investigating the percentage of time the channel is getting busy due to collisions. This is different from what Equation (13) reflects. Equation (13) describes the probability of collision from each node's perspective. In other words, this equation describes the average probability of collision that any node will face when communicating over the medium. However, that equation does not consider the channel's perspective. The latter recognizes the fact that a collision involves at least two nodes, and therefore, even if three or more nodes send their packets at the same instant, the channel will experience a busy period of only $L_{c}$ time units. Stated differently, by examining Fig. 4, we notice that the proportion of time a node spends in the collision state is $L_{c}(1-\alpha)(1-\beta) P_{c} \tau$ (recall Equation (11)). Then, if $N_{c}$ nodes (out of $N$ ) have collided at the same instant, the channel collision time, $T_{C C}$, is the non-overlapping period of time during which the channel is busy with a collision situation. The latter is defined as follows:

$T_{c c}=\frac{L_{c} N \tau(1-\alpha)(1-\beta) P_{C}}{N_{c}}$

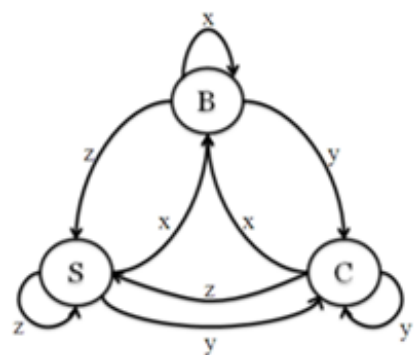

(a)

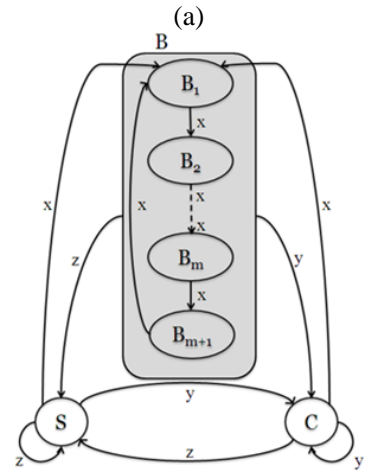

(b)

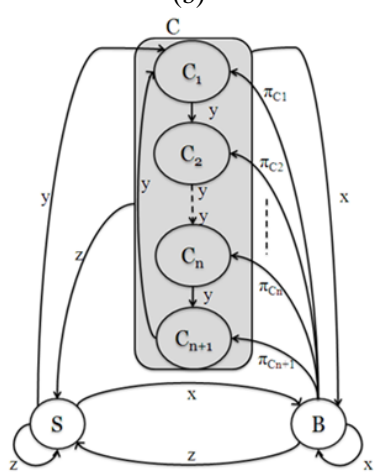

(c)

Fig. 5. (a) States Encountered During One Complete Cycle, (b) Break Down of the B State into Multiple Backoff Stages, and (c) Break Down of the C State into Multiple Packet Transmission Retries.

where, $N_{c}$ is the expected number of collided nodes. To determine the value of $N_{c}$, we should calculate the expected 
number of collided nodes, given that a collision has happened. This is a conditional probability that we compute as follows. The probability of having $k$ nodes involved in a collision requires that while a node is at CCA1, $k-1$ nodes should also be at CCA1 while the remaining $N-k$ nodes should be in any state other than CCA1. This probability is expressed as $\tau^{k-1}(1-\tau)^{N-k}$. The problem of selecting $k-1$ nodes out of $N$ 1 nodes under the conditions just mentioned is a typical binomial distribution. Given all of these facts, and by noticing that we may have from 2 to $\mathrm{N}$ nodes that are colliding at the same time, we can formulate $N_{c}$ as follows:

$N_{c}=\frac{1}{P_{c}} \sum_{k=2}^{N}\left(\begin{array}{c}k-1 \\ N-1\end{array}\right) k \tau^{k-1}(1-\tau)^{N-k}$

Note that we divide by Pc because we have a condition that a collision has already happened.

Equations (32) and (33) imply that if $k$ nodes have collided at the same time, the channel will be busy for only $L_{c}$ time units and not $k L_{c}$.

An effective backoff algorithm should be able to achieve reductions in $T_{C C}$.

\section{SimUlations AND MODEL VALIDATION}

In this section we conduct extensive simulations in order to validate the mathematical model developed in Section IV. Our simulations also provide a comparative study between ABA, from one side, and both BEB and NO-BEB from the other side. In this comparison, we evaluate the performance of ABA in terms of channel utilization, power consumption, reliability, and channel collision time. Furthermore, the fairness of ABA is studied to ensure that the nodes in the network are sharing the communication medium equally.

We wrote a C-based simulator to simulate ABA and the other three algorithms mentioned above. The network under study is of a peer-to-peer topology. The network operates in the beacon-enabled IEEE 802.15.4 mode. We omit both the CFP and the inactive periods from the superframe and assume that it is constituted only by the CAP in the active period.

We use the average power consumption of different wireless network interface cards (NICs) [60], [61], and [62]. The parameters considered in our simulations are summarized in Table $\mathrm{I}^{1}$. Also, we always assume, except when stated differently, that $L_{s}=L_{c}=L$. In the following sub-sections, we present our simulations results along with discussions and comments.

\section{A. Model Validation}

In this subsection we validate our theoretical Markov model by comparing the behavior it predicts to the behavior extracted from simulations. For each parameter studied, we compute the coefficient of variation of the root-mean-square deviation RSMD (CV(RMSD)), which is a measure of the accuracy of our mathematical model. In other words, $C V(R M S D)$ measures the differences between the

${ }^{1}$ CCA power in this table refers to the power consumed during either of the clear channel assessment periods. mathematical model and the simulations. $C V(R S M D)$ is defined as follows:

$C V(R S M D)=\frac{\sqrt{\frac{\sum_{i=1}^{n}\left(V_{\text {theo }}-V_{\text {sim }}\right)^{2}}{n_{\text {sample }}}}}{\bar{V}}$

where, $V_{\text {theo }}$ is the predicted theoretical value, $V_{\text {sim }}$ is the simulated value, $\bar{V}$ is the average of the observed values, and $n_{\text {sample }}$ is the total number of the sample values used. An accurate theoretical model should achieve low values for $C V$ (RMSD).

1) Channel utilization: We validate the mathematical expression that we derived for $U$ in Equation (19). Fig. 6 compares the theoretical behavior with the simulated behavior under unacknowledged traffic conditions while Fig. 7 shows the comparison under acknowledged traffic conditions. We can clearly see that Equation (19) is very accurate in predicting the behavior $U$ of as the network's size increases. We do see, however, a discrepancy for small networks $(\mathrm{N} \leq$ 20). In fact, we explain this discrepancy by recalling that Equations (16) and (18) are approximated for large N (see Section IV), and therefore, as the network gets smaller the model, we provided, may become less accurate.

TABLE I. SIMULATION PARAMETERS

\begin{tabular}{|l|l|l|}
\hline \multirow{4}{*}{$\begin{array}{l}\text { Power } \\
\text { Consumed } \\
(\mathbf{m W})\end{array}$} & $\mathrm{Rx}$ & 30 \\
\cline { 2 - 3 } & $\mathrm{Tx}$ & 40 \\
\cline { 2 - 3 } & $\mathrm{CCA}$ & 30 \\
\cline { 2 - 3 } & Sleep & 0.8 \\
\hline \multirow{4}{*}{\begin{tabular}{l} 
Durations \\
\cline { 2 - 3 }
\end{tabular}} & 1 timeslot & $0.32 \mathrm{~ms}(80$ bits $)$ \\
\cline { 2 - 3 } & Packet Length $(L)$ & 14 or 28 timeslots \\
\cline { 2 - 3 } $\begin{array}{l}\text { 802.15.4 } \\
\text { Parameter } \\
\text { Settings }\end{array}$ & ACK Packet Length $\left(L_{A C K}\right)$ & 2 timeslots \\
\cline { 2 - 3 } & Simulation Time & $320 \mathrm{~s}$ \\
\cline { 2 - 3 } & macMinBE & 3 \\
\hline
\end{tabular}

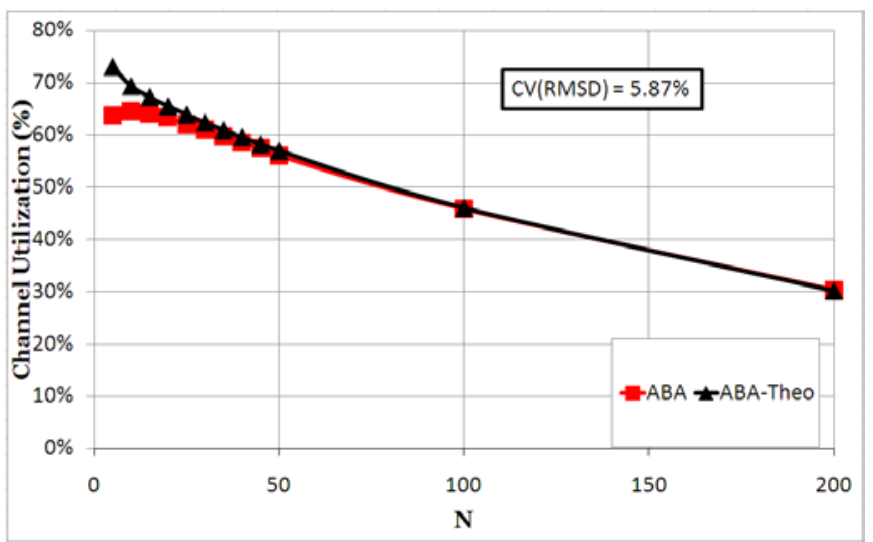

Fig. 6. Channel Utilization of ABA Under Unacknowledged Traffic. L=14, macMaxCSMABackoff=4, and macMaxFrameRetries=3. 


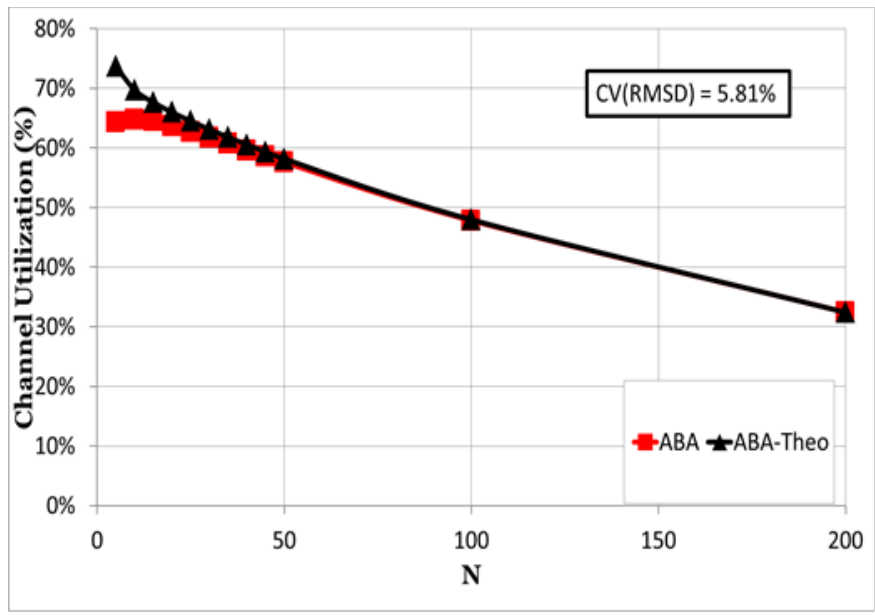

Fig. 7. Channel Utilization of ABA under Acknowledged Traffic. L=14, macMaxCSMABackoff=4, and macMaxFrameRetries=3.

2) Power consumption: Fig. 8, for unacknowledged traffic, and Fig. 9, for acknowledged traffic, show a perfect match between our mathematical expressions and the simulations for the total power consumption under ABA.

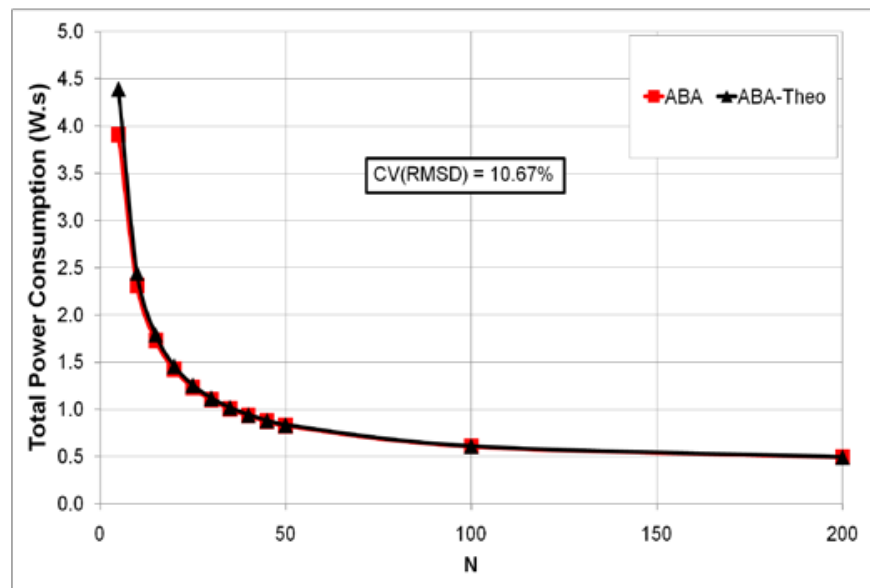

Fig. 8. Total Power Consumption (W.s) of ABA Under Unacknowledged Traffic. L=14, macMaxCSMABackoff=4, and macMaxFrameRetries=3.

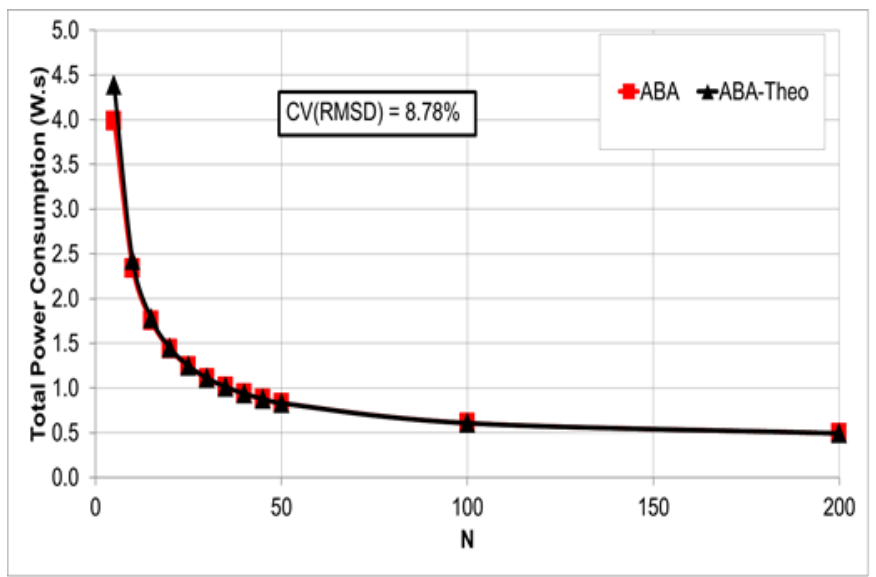

Fig. 9. Total Power Consumption (W.s) of ABA Under Acknowledged Traffic. $\mathrm{L}=14$, macMaxCSMABackoff $=4$, and macMaxFrameRetries=3.
3) Power wasted in collisions: Fig. 10, for unacknowledged traffic, and Fig. 11, for acknowledged traffic, depict the theoretical and simulated performance in terms of the power wasted due to packet collisions. These figures illustrate an accurate matching between our Markov model and the simulations.

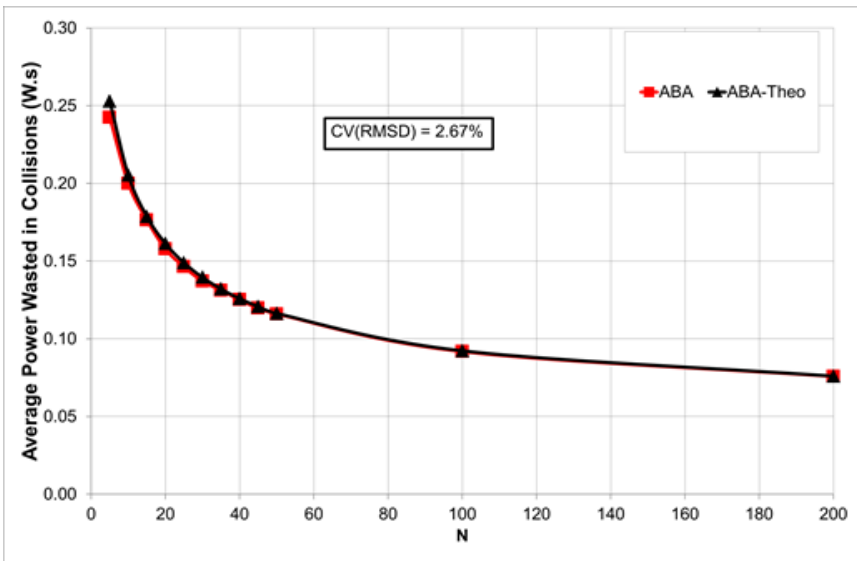

Fig. 10. Power Wasted In Collisions (W.s) Under Unacknowledged Traffic. $\mathrm{L}=14$, macMaxCSMABackoff $=4$, and macMaxFrameRetries $=3$.

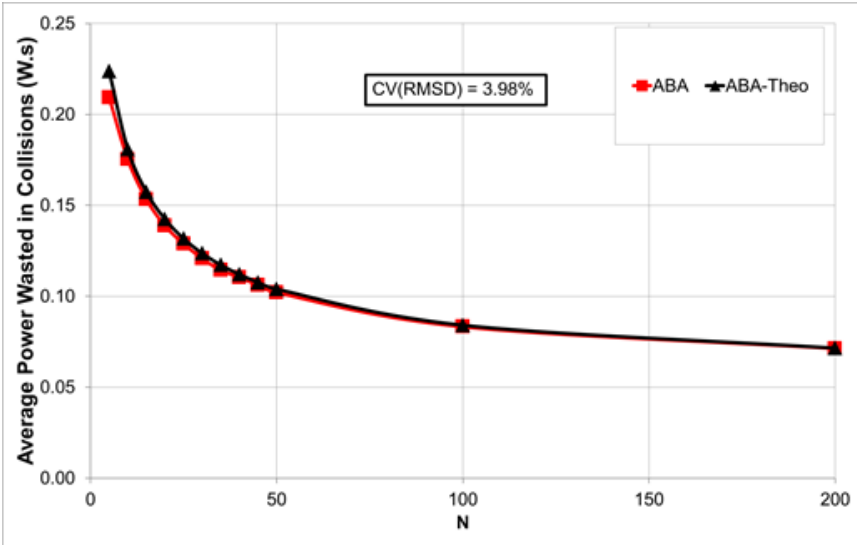

Fig. 11. Power Wasted In Collisions (W.s) Under Acknowledged Traffic. $\mathrm{L}=14$, macMaxCSMABackoff $=4$, and macMaxFrameRetries $=3$.

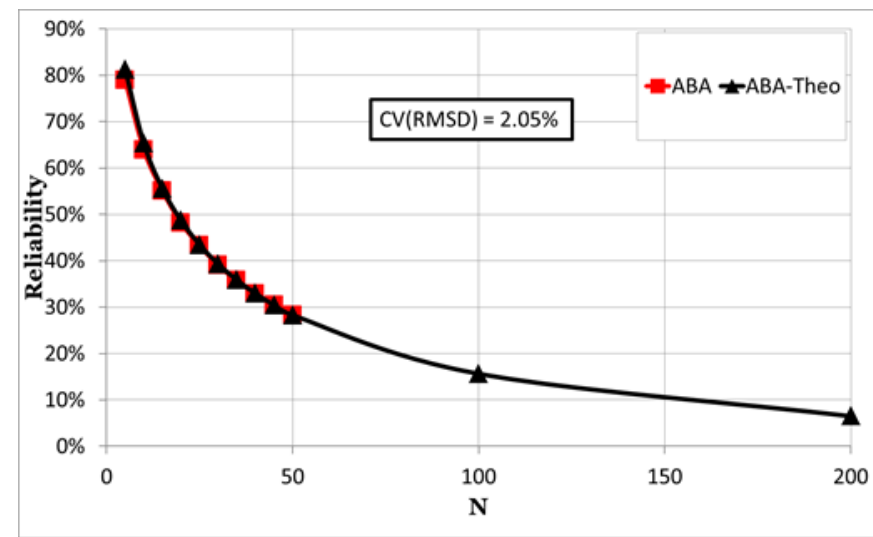

Fig. 12. Reliability of ABA Under Unacknowledged Traffic. L=14, macMaxCSMABackoff=4, and macMaxFrameRetries=3. 
4) Reliability: Fig. 12, for unacknowledged traffic, show a perfect match between our mathematical expression and the simulations for the reliability of ABA. The same observation is seen in Fig. 13 for the acknowledged traffic.

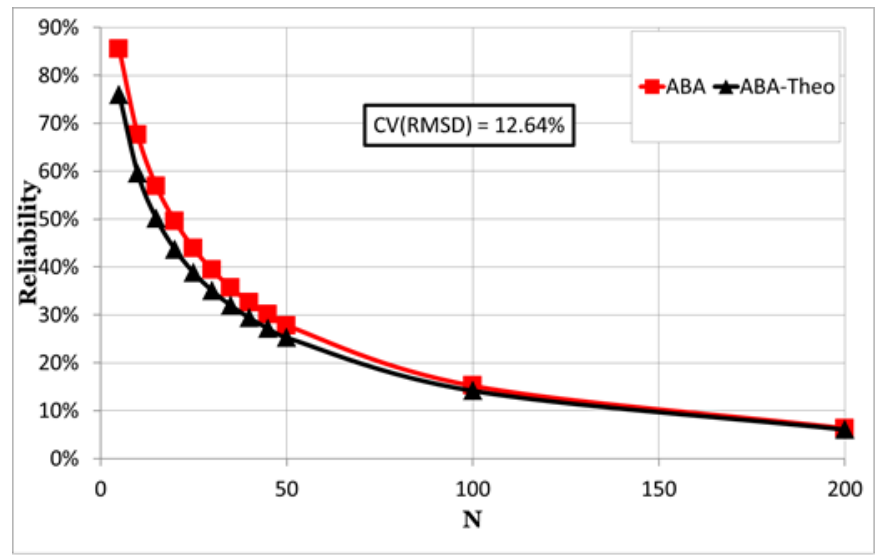

Fig. 13. Reliability of ABA Under Acknowledged Traffic. $L=14$, macMaxCSMABackoff=4, and macMaxFrameRetries=3.

5) Channel collision TimeL: ABA's theoretical and simulated behavior in terms of the achieved channel collision time, under different traffic conditions, is depicted in Fig. 14 and 15. Although we observe a deviation between the theoretical curves and the simulation ones in all of these figures, the deviation is minor and does not undermine the accuracy of our model. We argue, however, that this deviation is occurring as a result of the term $N_{c}$ in Equation (32). $N_{c}$ is computed using Equation (33), which includes the term $(1-\tau)^{N-k}$. We discussed in Section IV that this term, originally used in Equation (13), is formed based on the assumption that a node that is not at the CCA1 state can be at any other state in the Markov chain of Fig. 4. This assumption provides a reasonable approximation of the probability of collision in the network, and the deviations we see in Fig. 14 and 15 are resulting from it.

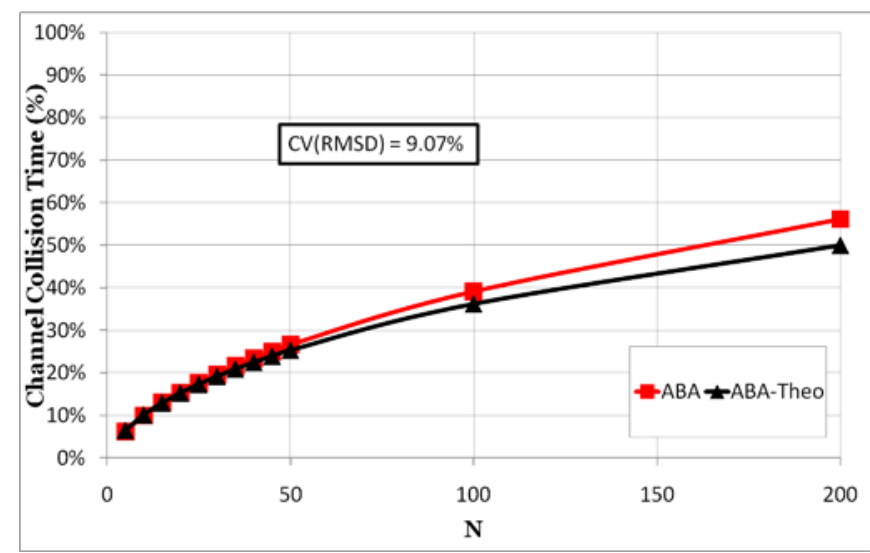

Fig. 14. Channel Collision Time with ABA, Under Unacknowledged Traffic. $\mathrm{L}=14$, macMaxCSMABackoff $=4$, and macMaxFrameRetries $=3$.

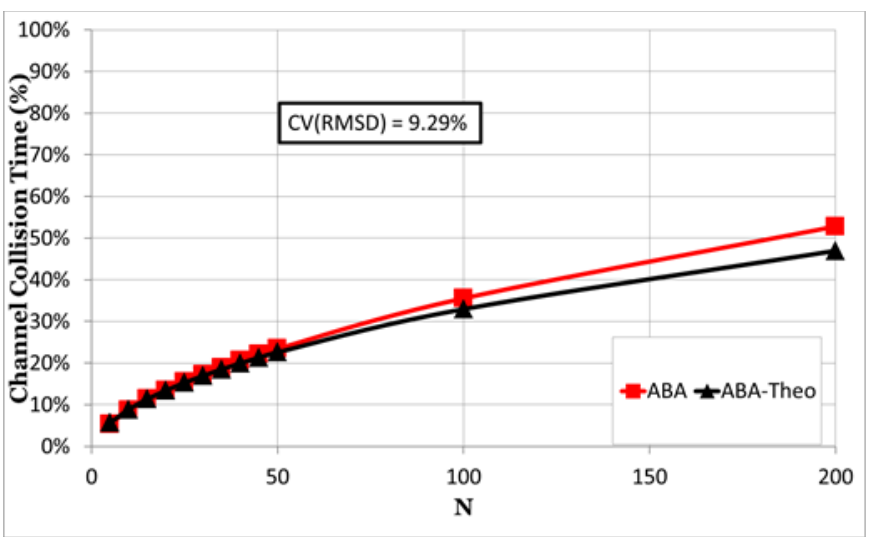

Fig. 15. Channel Collision Time with ABA, Under Acknowledged Traffic. $\mathrm{L}=14$, macMaxCSMABackoff $=4$, and macMaxFrameRetries=3.

In general, by examining Fig. 6 to 15, and by noticing the values of that we achieved, we can conclude that our Markovbased theoretical model of ABA is accurate and successful in predicting the simulated performance.

\section{B. Comparing ABA with other Algorithms}

In this subsection we study the performance of $A B A$ compared to that of NO-BEB and the standard BEB.

1) Channel utilization: We show in Fig. 16 ABA's performance in terms of channel utilization, under unacknowledged traffic conditions, compared to BEB and NO-BEB. The comparison under acknowledged traffic conditions is shown in Fig. 17. We can see in these figures that $\mathrm{ABA}$ achieves a superior performance compared to $\mathrm{BEB}$ and NO-BEB. The enhancements over thesse algorithms become significant as the network's size increases (especially beyond a size of 20 nodes). For example, in Fig. 16, at 35 nodes, ABA achieves a $U$ of $59.84 \%$, while BEB and NOBEB achieve $19.63 \%$ and $35.63 \%$, respectively. This means that as ABA enables nodes to update the size of their contention windows in an adaptive manner, a better utilization of the communication channel is achieved.

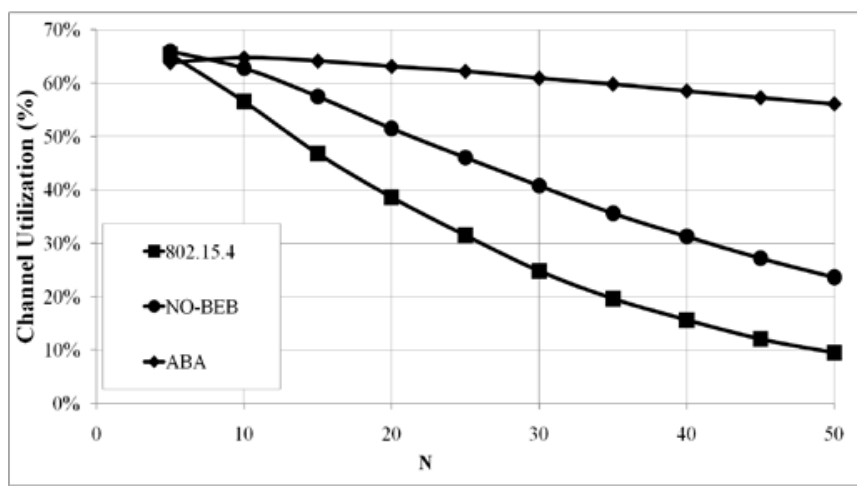

Fig. 16. Channel Utilization of ABA, BEB, and NO-BEB Under Unacknowledged Traffic. $\mathrm{L}=14$, macMaxCSMABackoff $=4$, and macMaxFrameRetries=3. 


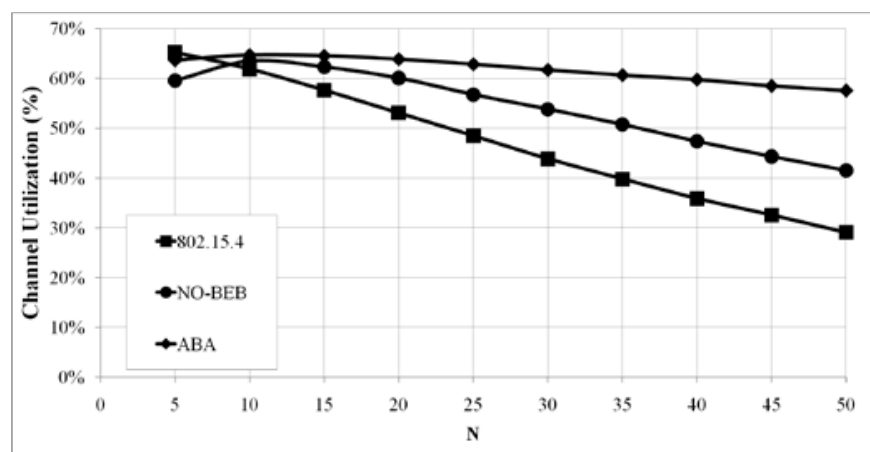

Fig. 17. Channel Utilization of ABA, BEB, and NO-BEB Under Acknowledged Traffic. $\mathrm{L}=14$, macMaxCSMABackoff $=4$, and macMaxFrameRetries=3.

2) Power consumption: In Fig. 18 and 19 we show the performance of $\mathrm{ABA}$ in terms of power consumption. It is evident in all these figures that $\mathrm{ABA}$ is consuming the least amount of power among all the algorithms. Compared to BEB (Fig. 18), ABA is able to achieve another $10.3 \%(a t ~ N=5)$ to $36.7 \%$ (at $\mathrm{N}=50$ ) of power savings. The savings compared to NO-BEB range from $3.5 \%$ to $21.8 \%$. Comparable results can be drawn from Fig. 19. The power savings achieved with ABA do not reflect a strong performance boost, and therefore, we need to investigate the portion of the total power that is wasted in useless activities, that is, collisions. In Fig. 20 and 21 we show the amount of power lost due to collisions under each algorithm. It its quite evident that $A B A$ is capable of lowering the percentage of collisions, and therefore, the power lost during these situations is the lowest compared to the other algorithms. In Fig. 20, compared to BEB, ABA manages to reduce the power wasted due to collisions significantly. At $\mathrm{N}$ $=5, \mathrm{ABA}$ wastes $39 \%$ less in power than BEB. At $\mathrm{N}=50$, the power wasted is $69.3 \%$ less than BEB. That is, ABA is able is to utilize the power resources of the sensor nodes in useful activities. Compared to NO-BEB, ABA loses less in power by $16.7 \%$ due to collisions ( $\mathrm{At} \mathrm{N}=5$ ). The savings in power jump to $50.6 \%$ at $\mathrm{N}=50$. Comparable conclusions can be observed in Fig. 21. In conclusion, we can see that ABA is proving to be more conservative in depleting the power resources of the nodes.

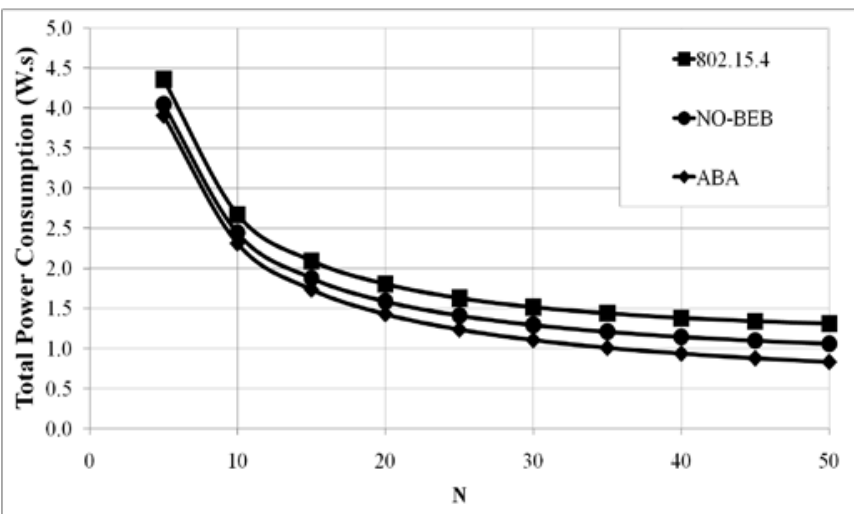

Fig. 18. Total Power Consumption (W.s) of ABA, BEB, and NO-BEB Under Unacknowledged Traffic. $\mathrm{L}=14$, macMaxCSMABackoff $=4$, and macMaxFrameRetries=3.

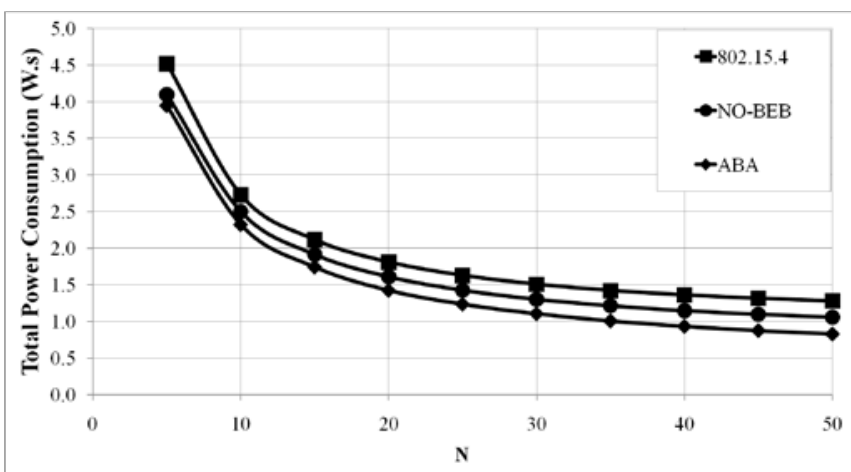

Fig. 19. Total Power Consumption (W.s) of ABA, BEB, and NO-BEB Under Acknowledged Traffic. $\mathrm{L}=14$, macMaxCSMABackoff $=4$, and macMaxFrameRetries=3.

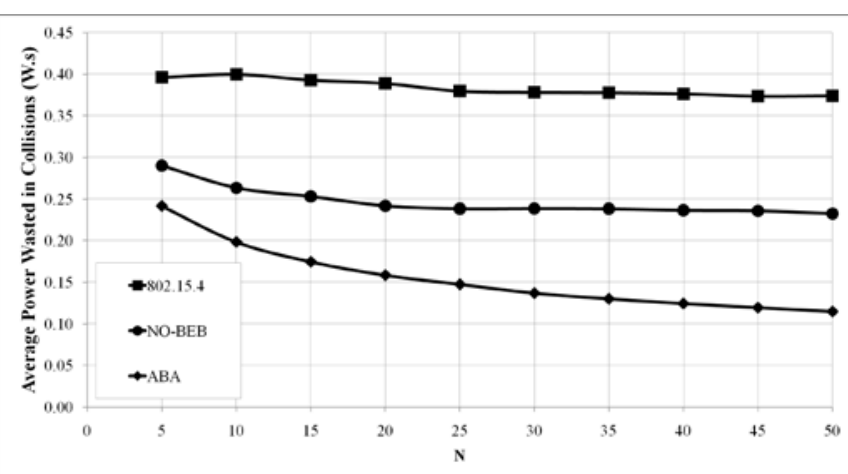

Fig. 20. Power Wasted in Collisions (W.s) Under Unacknowledged Traffic. $\mathrm{L}=14$, macMaxCSMABackoff=4, and macMaxFrameRetries=3.

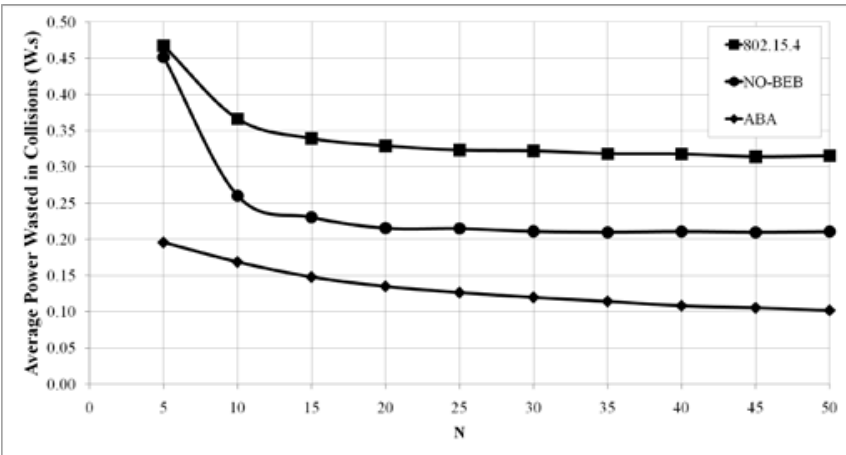

Fig. 21. Power Wasted in Collisions (W.s) Under Acknowledged Traffic. $\mathrm{L}=14$, macMaxCSMABackoff $=4$, and macMaxFrameRetries $=3$.

3) Reliability: In Fig. 22 and 23 we depict the performance of ABA in terms of the reliability. These figures demonstrate the superiority of $\mathrm{ABA}$ over all the other algorithms in terms of reliability. A significant improvement can be observed over BEB and NO-BEB. In particular, in Fig. 22, ABA manages to achieve a boost in reliability that starts from 39\% (at $\mathrm{N}=5$ ) and keeps increasing till 69.3\% (at $\mathrm{N}=50$ ) compared to BEB. Compared to NO-BEB, the increase in reliability goes from $8.32 \%$ (at $\mathrm{N}=10$ ) and continues to $61.8 \%$ (at $\mathrm{N}=50$ ). In Fig. 23, we can observe a similar performance. 


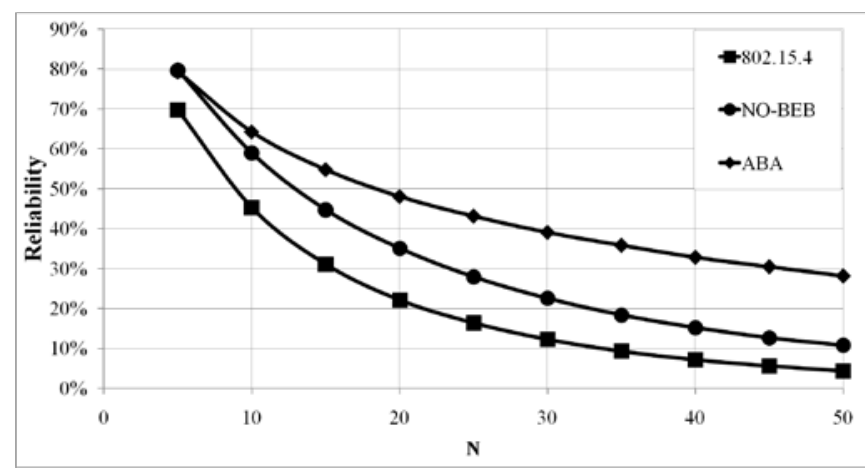

Fig. 22. Reliability of ABA Under Unacknowledged Traffic. $L=14$, macMaxCSMABackoff=4, and macMaxFrameRetries=3.

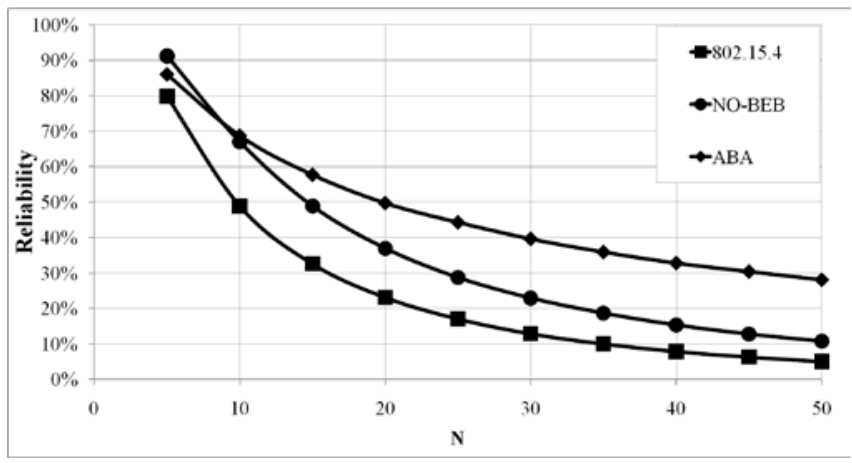

Fig. 23. Reliability of ABA Under Acknowledged Traffic. $L=14$, macMaxCSMABackoff=4, and macMaxFrameRetries=3.

4) Channel collision time: We examine the performance in terms of the channel collision time in Fig. 24 and 25. Again, ABA is showing superiority in terms of its ability to keep the channel collision time at its lowest level compared to the other algorithms. In particular, in Fig. 24, ABA can achieve a channel collision time that is $38.5 \%$ less than $\mathrm{BEB}$ at $\mathrm{N}=5$. This percentage jumps to $58.8 \%$ at $\mathrm{N}=50$. Compared to NO$\mathrm{BEB}, \mathrm{ABA}$ 's improvements in channel collision time range from $16.5 \%$ (at $\mathrm{N}=5$ ) and $43.74 \%$ (at $\mathrm{N}=50$ ). Again, comparable observations can be seen in Fig. 25. ABA's ability to adapt the contention window's size in accordance with the collisions level allows for an efficient utilization of the network's resources.

5) Fairness: Finally, we examine the fairness of $A B A$ in order to see whether it allows nodes an equal opportunity to access the wireless medium or not. We adopt Jain's fairness index [40] to measure ABA's fairness:

fairness index $=\frac{\left(\sum x_{i}\right)^{2}}{N \sum x_{i}{ }^{2}}$

where, $x_{i}$ denotes the $i$ th node's share of the medium. An algorithm is achieving better sharing of the medium among the nodes if its fairness index is closer to 1 .

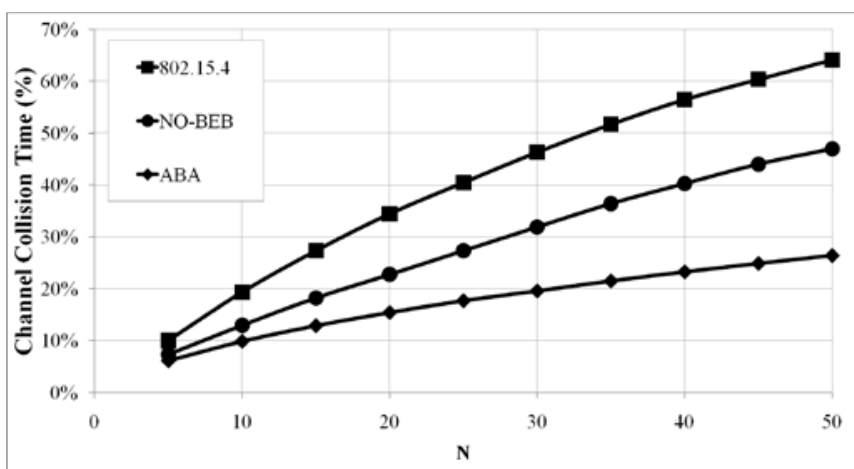

Fig. 24. Channel Collision Time with ABA, Under Unacknowledged Traffic. $\mathrm{L}=14$, macMaxCSMABackoff $=4$, and macMaxFrameRetries=3.

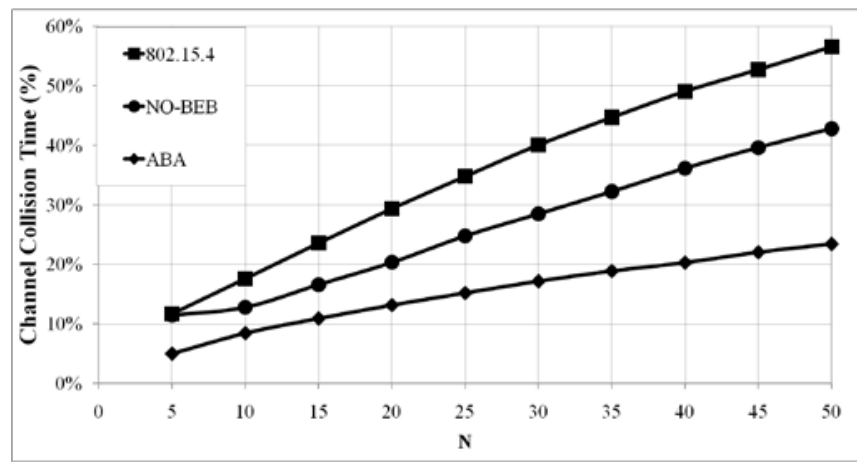

Fig. 25. Channel Collision Time with ABA, Under Acknowledged Traffic. $\mathrm{L}=14$, macMaxCSMABackoff $=4$, and macMaxFrameRetries $=3$.

Fig. 26 shows the fairness of ABA, BEB, and NO-BEB under unacknowledged traffic conditions while Fig. 27 shows the fairness under acknowledged traffic conditions. We can clearly see that, for different packet lengths, $A B A, B E B$, and NO-BEB achieve a fair sharing of the medium among the nodes (the three curves are overlapping, and therefore, only one curve is apparent in the figures).

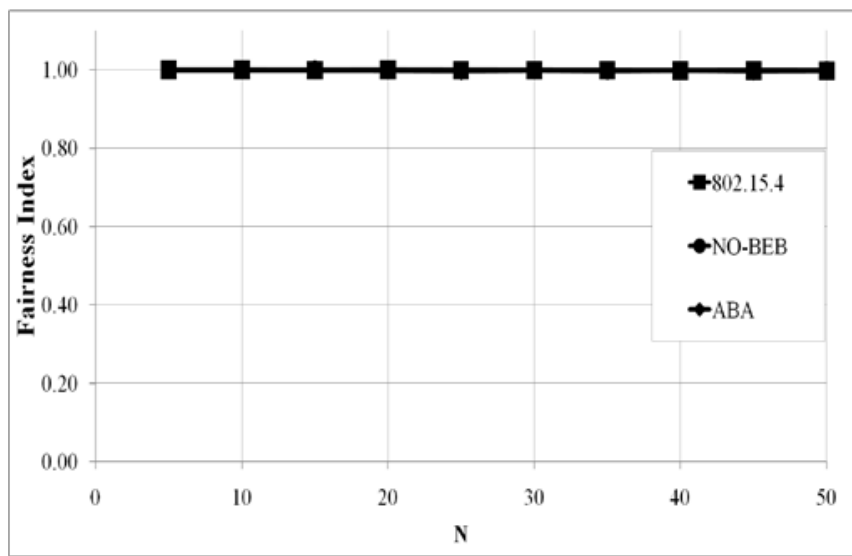

Fig. 26. Fairness of ABA Under Unacknowledged Traffic. $L=14$, macMaxCSMABackoff=4, and macMaxFrameRetries=3. 


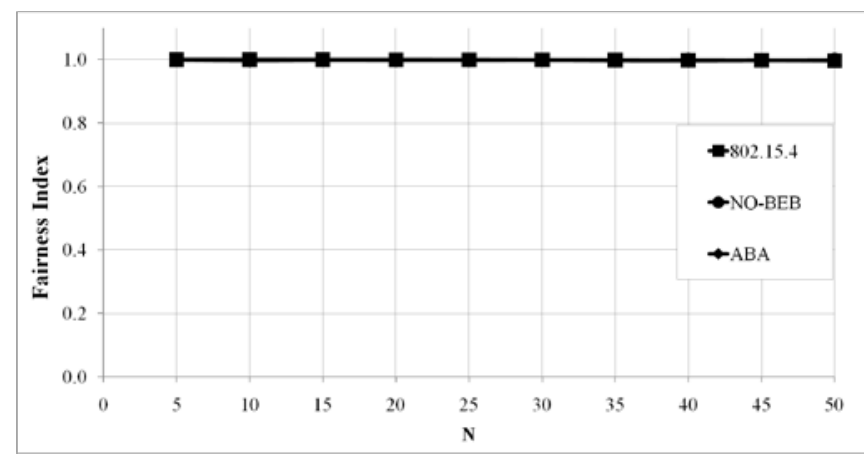

Fig. 27. Fairness of ABA Under Acknowledged Traffic. $L=14$, macMaxCSMABackoff $=4$, and macMaxFrameRetries $=3$.

\section{Discussions}

In the previous subsection we found that the $\mathrm{ABA}$ algorithm is achieving a promising performance in terms of enhancing channel utilization, conserving power resources, improving reliability, reducing the level of collisions, while preserving the fairness among the nodes in the network. The superiority of ABA over the other algorithms, especially BEB, comes from the fact that it indirectly relates the contention window $(W)$ to the number of nodes in the network. This can be understood by recalling Equation (13) in which we have a direct relation between the probability of collision $\left(P_{c}\right)$ and the number of nodes in the network $(N) . P_{c}$ increases with the increase of $N$, and therefore, the value of the $W$ should be changed taking into consideration the size of the network. Thus, the main strength of ABA is that it is controlling $W$ probabilistically, in such a way it self-adapts to the network's size and the activity over the communication channel. Depending on a deterministic methodology, as in BEB, to change $W$ without any consideration for the network's size gives a poor performance as we demonstrated. The same problem is found with NO-BEB which adopts from BEB the idea of resetting $W$ to a predefined minimum. Although NOBEB shows a considerable improvement over BEB's performance, resetting $W$ to its minimum without taking into consideration the current status over the medium will degrade the performance.

\section{CONCLUSION AND FUTURE DIRECTIONS}

In this paper we studied IEEE 802.15.4's BEB algorithm and highlighted its major limitations that degrade the overall performance of the wireless sensor network. We pointed out that BEB's methodology of updating the size of the contention window is highly deterministic and cannot cope with the changing levels of activity over the communication channel. Based on these observations, we introduced a novel backoff algorithm, the Adaptive Backoff Algorithm (ABA), which introduces an adaptive, probabilistic methodology to control the size of the contention window. ABA depends on including the probability of collisions, as computed locally by each sensor node, in the computation of the size of the contention window. In this way, the number of nodes competing to access the medium is involved indirectly in the process of updating the contention window. Therefore, we end up with a backoff algorithm that self-adapts to the size of the network, and therefore, manages the medium access in a way that improves the overall performance. We modeled ABA using Markov chain and validated our model using a C-based simulator. Our simulations prove the accuracy of our theoretical model of ABA. Also, they demonstrated a superior performance over BEB, as well as two other backoff algorithms, in terms of channel utilization, power consumption, reliability, and channel collision time. The simulations also proved that ABA is fair in terms of allowing the competing nodes an equal opportunity to access the medium.

As future research directions, we will work on elaborating the concept of probabilistic backoff algorithms such that more effective MAC protocols are designed for wireless sensor networks. In fact, the methodology of exploiting the probability of collision to gain information about the size of the network and the status over the communication medium can prove usefulness in prioritizing nodes' access to the access the medium. We plan to explore the latter topic in our future research.

\section{REFERENCES}

[1] K. J. Son, S. H. Hong, S. P. Moon, T. G. Chang and H. Cho, "Segmentized clear channel assessment for IEEE 802.15." 4 networks. Sensors, 16(6), 815, 2016.

[2] T. M. Hoang, V. T. Nguyen, N. G. Nguyen and T. N. Lang, "Analysing the performance of unslotted sensor networks based on the IEEE 802.15. 4 employed EIED algorithm.” In 2017 International Conference on Information Networking (ICOIN) (pp. 682-685). IEEE, 2017, January.

[3] S. Xie, K. S. Low and E. Gunawan, "A distributed transmission rate adjustment algorithm in heterogeneous CSMA/CA networks." Sensors, 15(4), 7434-7453, 2015.

[4] H. R. Hussen, C. R. Teja, T. Miao, K. Kim and K. H. Kim, "Trafficaware cooperative binary exponential backoff algorithm for low power and lossy networks." Wireless Personal Communications, 86(4), 19131929, 2016.

[5] H. P. Sultana and P. V. Krishna, "Priority focused medium access control in wireless sensor actuator networks for CPS.” International Journal of Communication Networks and Distributed Systems, 16(2), 99-113, 2016.

[6] Z. Yifan, S. Zhou, H. Ding, Z. Yang and Q. Liu, "IEEE 802.15. 4 CSMA/CA Scheme for Heterogeneous Sensor Networks Based Adaption and RTS/CTS Mechanism.” Sensor Letters, 14(7), 719-726, 2016.

[7] M. Elappila, S. Chinara and D. R. Parhi, "Survivability Aware Channel Allocation in WSN for IoT applications." Pervasive and Mobile Computing, 61, 101107, 2020.

[8] Mounib Khanafer, Mouhcine Guennoun, Hussein T. Mouftah, An Efficient Adaptive Backoff Algorithm for Wireless Sensor Networks, IEEE Global Communications Conference, GLOBECOM 2011, Houston, Texas, USA, 5-9 December 2011.

[9] F. I. Engoti, "Réalisation d'une plate-forme pour l'optimisation de réseaux de capteurs sans fil appliqués au bâtiment intelligent” (Doctoral dissertation, Limoges), 2018.

[10] M. G. Asuti and P. I. Basarkod, "An optimal clear channel assessment in IEEE 802.15. 4 medium access control protocol for recurrent data transmission and long acknowledgement wait period.” Transactions on Emerging Telecommunications Technologies, e4167, 2020.

[11] M. Li, J. Dong, Y. Zhang, H. Yang, L. V. Zwieten, H. Lu, ... and X. Jiang, "A Critical Review of Methods for Analyzing Freshwater Eutrophication.” Water 2021, 13, 225, 2021.

[12] P. S. Habibullah and N. Nagendhiran, "Medium Access Control Methods in Sensor and Actuator Based Wireless Networks-A Review.” Walailak Journal of Science and Technology (WJST), 14(4), 267-274, 2017.

[13] M. Khanafer, M. Guennoun and H. T. Mouftah, "Priority-Based CCA Periods for Efficient and Reliable Communications in Wireless Sensor 
Networks,” Wireless Sensor Network (WSN), Scientific Research, Vol. 4, No. 2, February 2012.

[14] B. Bala, M. Pandey and D. Prasad, "An Adaptive Timeslot Allocation Scheme for Wireless Body Area Networks." International Journal of Computer Applications, 975, 8887, 2016.

[15] B. Bala and M. Pandey, "Survey on Priority Based Schemes Used For Data Dissemination in Wireless Body Area Network." International Journal of Advanced Research in Computer Science, 6(2), 2015.

[16] M. Gamal, N. Sadek, M. Rizk and M. Ahmed, "Markov Model of Modified Unslotted CSMA/CA for Wireless Sensor Networks.” In 2019 31st International Conference on Microelectronics (ICM) (pp. 57-61). IEEE, 2019, December.

[17] P. K. Sahoo, S. R. Pattanaik and S. L. Wu, "A reliable data transmission model for ieee 802.15. 4e enabled wireless sensor network under wifi interference.” Sensors, 17(6), 1320, 2017.

[18] Hussein T. Mouftah, Mounib Khanafer, Mouhcine Guennoun, Wireless sensor network architectures for intelligent vehicular systems, Symposium International for Telecommunication Techniques, 2010.

[19] P. K. Sahoo, S. R. Pattanaik and S. L. Wu, "A novel IEEE 802.15. 4e DSME MAC for wireless sensor networks.” Sensors, 17(1), 168, 2017.

[20] M. Gamal, N. Sadek, M. R. Rizk and M. A. E. Ahmed, "Optimization and modeling of modified unslotted CSMA/CA for wireless sensor networks.” Alexandria Engineering Journal, 59(2), 681-691, 2020.

[21] G. Bianchi, "Analysis of the IEEE 802.11 distributed coordination function," In IEEE Journal on Selected Areas in Communications, vol. 18, no. 3, pp. 535-547, Mar. 2000.

[22] G. Bianchi, L. Fratta and M. Oliveri, "Performance Evaluation and Enhancement of the CSMA/CA MAC Protocol for 802.11 Wireless LANs," in proceedings of the IEEE International Symposium on Personal, Indoor and Mobile Radio Communications (PIMRC'96), pp. 392-396, Taipei, Taiwan, Oct. 1996.

[23] F. Calì, M. Conti and E. Gregori, "Dynamic Tuning of the IEEE 802.11 Protocol to Achieve a Theoretical Throughput Limit," IEEE/ACM Transactions on Networking, vol. 8, no. 6, Dec. 2000.

[24] M. Albalt and N. Qasim, "Adaptive Backoff Algorithm for IEEE 802.11 MAC Protocol,” International Journal of Communications, Network and System Sciences, vol. 2, no. 4, pp. 249-324, Jul. 2009.

[25] V. V. Kamath, "An Approach to Increase Channel Utilization in the IEEE 802.11 Networks by Improving Fairness at the Medium Access Control Sub-Layer,” Master's thesis, George Mason University, Dec. 2008.

[26] S. Xu and T. Saadawi, "Does the IEEE 802.11 MAC protocol work well in multihop wireless ad hoc networks?,” IEEE Communications Magazine, vol. 39. no. 6, pp. 130-137, Jun. 2001.

[27] N. O. Song, B. J. Kwak, J. Song and L. E. Miller, "Enhancement of IEEE 802.11 Distributed Coordination Function with Exponential Increase Exponential Decrease Backoff Algorithm," in proceedings of the $57^{\text {th }}$ IEEE Semiannual Vehicular Technology Conference (VTC’03), vol. 4, pp. 2775-2778, Apr. 2003.

[28] J. G. Ko, Y. H. Cho and H. Kim, "Performance Evaluation of IEEE 802.15.4 MAC with Different Backoff Ranges in Wireless Sensor Networks," in proceedings of the $10^{\text {th }}$ IEEE International Conference on Communications Systems (ICCS’06), pp. 1-5, Singapore, Oct. 2006.

[29] S. Y. Lee, Y. S. Shin, J. S. Ahn and K. W. Lee, "Performance Analysis of a Non-Overlapping Binary Exponential Backoff Algorithm over IEEE 802.15.4," in proceedings of the 4th International Conference on Ubiquitous Information Technologies \& Applications (ICUT’09), Japan, Dec. 2009.

[30] S. Woo, W. Park, S. Y. Ahn, S. An and D. Kim, "Knowledge-Based Exponential Backoff Scheme in IEEE 802.15.4 MAC," Lecture Notes in Computer Science (LNCS), vol 5200, pp. 435-444, 2008.

[31] B. M. Khan, F. H. Ali and E. Stipidis, "Improved Backoff Algorithm for IEEE 802.15.4 Wireless Sensor Networks," in proceedings of the $3^{\text {rd }}$ IFIP Wireless Days (WD’10), Italy, Oct. 2010.

[32] IEEE Std 802.15.4-2006, September, Part 15.4: Wireless Medium Access Control (MAC) and Physical Layer (PHY) Specifications for Low-Rate Wireless Personal Area Networks (WPANs).
[33] B. H. Lee and H. K. Wu, "Study on Backoff Algorithm for IEEE 802.15.4 LR-WPAN," in proceedings of the $22^{\text {nd }}$ International Conference on Advanced Information Networking and Applications (AINA'08), pp. 403 - 409, Okinawa, Japan, Mar. 2008.

[34] H. Minooei and H. Nojumi, "Performance evaluation of a new backoff method for IEEE 802.11”, Computer Communications, vol. 30, no. 18, pp. 3698-3704, Dec. 2007.

[35] Mounib Khanafer, Mouhcine Guennoun, Hussein T. Mouftah, Adaptive Sleeping Periods in Slotted IEEE 802.15.4 for Efficient Energy Savings: Markov-Based Theoretical Analysis, IEEE International Conference on Communications, ICC 2011, Kyoto, Japan, 5-9 June 2011.

[36] P. Park, P. D. Marco, C. Fischione and K. H. Johansson, "Adaptive IEEE 802.15.4 protocol for reliable and timely communications," in proceedings of the $9^{\text {th }}$ ACM/IEEE International Conference on Information Processing in Sensor Networks (IPSN'10), pp. 327-338, Stockholm, Sweden, Apr. 2010.

[37] S. Pollin, M. Ergen, S. C. Ergen, B. Bougard, L. V. derPerre, I. Moerman, A. Bahai, P. Varaiya and F. Catthoor, "Performance Analysis of Slotted Carrier Sense IEEE802.15.4 Medium Access Layer,” IEEE Transactions onWireless Communications, vol. 7, no. 9, pp. 3359-3371, Sept. 2008.

[38] J. Mišić and V. B. Mišić, "Access Delay for Nodes with Finite Buffers in IEEE 802.15.4 Beacon Enabled PAN with Uplink Transmissions," Computer Communications, vol. 28, no. 10, pp. 1152-1166, Jun. 2005.

[39] J. Zhu, Z. Tao and C. Lv, "Performance Evaluation of IEEE 802.15.4 CSMA/CA Scheme Adopting a Modified LIB Model," Wireless Personal Communications, Online First: http://www.springerlink.com/content/712431107jwx1h66/fulltext.pdf, pp. 1-27, Jan. 2011.

[40] R. Jain, D. Chiu and W. Hawe, "A Quantitative Measure of Fairness and Discrimination for Resource Allocation in Shared Computer Systems", DEC-TR-301, Sept. 26 $6^{\text {th }}, 1984$.

[41] M. Martalò, G. Ferrari and S. Busanelli, "Markov Chain-Based Performance Analysis of Multihop IEEE 802.15.4 Wireless Networks," Performance Evaluation, vol. 66, no. 12, pp. 722-741, Dec. 2009.

[42] I. Ramachandran, A. K. Das and S. Roy, "Analysis of the Contention Access Period of IEEE 802.15.4 MAC,” ACM Transactions on Sensor Networks, vol. 3, no. 1, article 4, Mar. 2007.

[43] Z. Xiao, C. He and L. Jiang, "Slot-Based Model for IEEE 802.15.4 MAC with Sleep Mechanism,” IEEE Communications Letters, vol. 14, no. 2, pp. 154-156, Feb. 2010.

[44] C. Y. Jung, H. Y. Hwang, D. K. Sung and G. U. Hwang, "Enhanced Markov Chain Model and Throughput Analysis of the Slotted CSMA/CA for IEEE 802.15.4 Under Unsaturated Traffic Conditions," IEEE Transactions on Vehicular Technology, vol. 58, no. 1, pp. 473478, Jan 2009.

[45] S. Fang, L. Rong, Q. Xu and Y. Du, "Analysis of Performance of Unsaturated Slotted IEEE 802.15.4 Medium Access Layer," in proceedings of PIERS, pp. 348-352, Beijing, China, Mar. 23-27, 2009.

[46] Z. Chen, C. Lin, H. Wen and H. Yin, "An Analytical Model for Evaluating IEEE 802.15.4 CSMA/CA Protocol in Low-Rate Wireless Application," in proceedings of the $21^{\text {st }}$ International Conference on Advanced Networking and Applications Wokshops (AINAW'07), vol. 2, pp. 899-904, Niagara Falls, Ontario, Canada, May 2007.

[47] I. Ramachandran, A. K. Das and S. Roy, "Analysis of the Contention Access Period of IEEE 802.15.4 MAC,” ACM Transactions on Sensor Networks, vol. 3, no. 1, article 4, Mar. 2007.

[48] K. Ashrafuzzaman and K. S. Kwak, "On the Performance Analysis of the Contention Access Period of IEEE 802.15.4 MAC," IEEE Communications Letters, vol. 15, no. 9, Sept. 2011.

[49] J. Zhu, Z. Tao and C. Lv, "Delay Analysis for IEEE 802.15.4 CSMA/CA Schemewith Heterogeneous Buffered Traffic," in proceedings of the $3^{\text {rd }}$ International Conference on Measuring Technology and Mechatronics Automation (ICMTMA'11), vol. 1, pp. 835-845, Shangshai, China, Jan. 2011.

[50] A. Faridi, M. R. Palattella, A. Lozano, M. Dohler, G. Boggia, L. A. Grieco and P. Camarda, "Comprehensive Evaluation of the IEEE 802.15.4 MAC Layer Performance With Retransmissions,” IEEE Transactions on Vehicular Technology, vol. 59, no. 8, Oct. 2010. 
[51] S. Wijetunge, U. Gunawardana and R. Liyanapathirana, "Performance Analysis of IEEE 802.15.4 MAC Protocol for WSNs with ACK Frame Transmission Under Unsaturated Traffic Conditions," in proceedings of the $6^{\text {th }}$ International Conference on Intelligent Sensors, Sensor Networks and Information Processing (ISSNIP'10), pp. 55-60, Brisbane, Australia, Dec. 2010.

[52] C. M. Wong, R. L. Lai and I. T. Lai, "An Enhanced Carrier Sensing Algorithm for IEEE 802.15.4 Low-Rate Wireless Sensor Networks," in proceedings of IEEE Symposium on Industrial Electronics and Applications (ISIEA'10), pp. 10-15, Penang, Malaysia, Oct. 2010.

[53] J. Deng, P. K. Varshney and Z. J. Hass, "A New Backoff Algorithm for the IEEE 802.11 Distributed Coordination Function," in proceedings of the Communication Networks and Distributed Systems Modeling and Simulation Conference (CNDS '04), San Diego, California, USA, Jan. 2004.

[54] K. C. Noh, S. Y. Lee, Y. S. Shin, K. W. Lee and J. S. Ahn, "Performance Evaluation of an Adaptive Congestion Avoidance Algorithm for IEEE 802.15.4,"in proceedings of the IEEE $13^{\text {th }}$ International Conference on Computational Science and Engineering (CSE'10), pp. 14-19, Hong Kong, China, Dec. 2010.

[55] P. Serrano, A. Banchs, V. Targon and J. F. Kukielka, "Detecting Selfish Configurations in 802.11 WLANs," IEEE Communications Letters, vol. 14, no. 2, pp. 142-144, Feb. 2010.

[56] L. Guang and C. Assi, "Mitigating Smart Selfish MAC Layer Misbehaviour in Ad Hoc Networks," in proceedings of IEEE International Conference on Wireless and Mobile Computing, Networking, and Communications (WiMob '06), pp. 116-123, Montreal, Quebec, Canada, Jun. 2006.

[57] L. Guang and C. Assi, "MAC Layer Misbehavior in Wireless Networks: Challenges and Solutions," IEEE Wireless Communications, vol. 15, no. 4, pp. 6-14, Aug. 2008.

[58] M. Raya, I. Aad, J. P. Hubaux and A. El Fawal, "DOMINO: Detecting MAC Layer Greedy Behaviour in IEEE 802.11 Hotspots,” IEEE Transactions on Mobile Computing, vol. 5, no. 12, pp. 1691-1705, Dec. 2006.

[59] K. Kredo and P. Mohapatra, "Medium access control in wireless sensor networks", Computer Networks: The International Journal of Computer and Telecommunications Networking, vol. 51, no. 4, pp. 961-994, 2007.

[60] Zolertia Z1 datasheet, http://zolertia.sourceforge.net/wiki/images/e/e8/ Z1_RevC_Datasheet.pdf, Mar. 2010.

[61] Lucent Technologies, WaveLAN/EC-S User's Guide, http://wireless.ictp.it/school_2001/docs/specs/orinoco/station_adapter.pd f.

[62] RFM ZPM3570 ZigBee Pro Module datasheet, http://www.rfm.com/products/data/zpm3570-e.pdf, May 2011.

[63] M. O. Rahman, C. S. Hong, S. Lee and Y. C. Bang, “ATLAS: A Traffic Load Aware Sensor MAC Design for Collaborative Body Area Sensor Networks,” Sensors, vol. 11, no. 12, pp. 11560-11580, Dec. 2011.

[64] B. Otal, L. Alonso and C. Verikoukis, "Energy-Efficiency Analysis of a Distributed Queuing Medium Access Control Protocol for Biomedical Wireless Sensor Networks in Saturation Conditions," Sensors, vol. 11, no. 2, pp. 1277-1296, Jan. 2011.

[65] X. Sun and L. Dai, "Backoff Design for IEEE 802.11 DCF Networks: Fundamental Tradeoff and Design Criterion," In Proceedings of the IEEE/ACM Transactions on Networking, Vol. 23, No. 1, pp. 300-316, 2015.

[66] D. Sharma, R. Srivastava and R. K. Sharma, "Effect of Contention Windows Size in Binary Exponential Back of Algorithm," in Proceedings of the Second International Conference on Advances in Computing and Communication Engineering, pp. 655-660, 2015.

[67] A. Ullah and J. S. Ahn, "Performance evaluation of X-MAC/BEB protocol for wireless sensor networks," In Journal of Communications and Networks, Vol. 18, No. 5, pp. 857-869, 2016.

[68] Q. Liu and A. Czylwik, "A collision-aware backoff mechanism for IEEE 802.15.4 wireless sensor networks," in 2013 IFIP Wireless Days (WD), pp. 1-3, 2013.

[69] M. Shurman, B. Al-Shua'b, M. Alsaedeen, M. F. Al-Mistarihi and K. A. Darabkh, "N-BEB: New backoff algorithm for IEEE 802.11 MAC protocol," In Proceedings of the 37th International Convention on
Information and Communication Technology, Electronics and Microelectronics, pp. 540-544, 2014.

[70] X. Liu, G. Ma, H. Kuang and F. Li, "An efficient backoff algorithm for QoS guaranteeing in wireless networks," In Proceedings of the Chinese Control and Decision Conference, pp. 5353-5358, 2016.

[71] Y. He, J. Sun, X. Ma, A. V. Vasilakos, R. Yuan and W. Gong, "SemiRandom Backoff: Towards Resource Reservation for Channel Access in Wireless LANs," In IEEE/ACM Transactions on Networking, Vol. 21, No. 1, pp. 204-217, Feb. 2013.

[72] M. A. Bender, J. T. Fineman, S. Gilbert and M. Young, "How to Scale Exponential Backoff: Constant Throughput, Polylog Access Attempts, and Robustness," In Proceedings of the Twenty-seventh Annual ACMSIAM Symposium on Discrete Algorithms, pp. 636-654, 2016.

[73] T. N. V. L. Alekhya, B. Mounika, E. Jyothi and B. N. Bhandari, "A waiting-time based backoff algorithm in the IEEE 802.11 based wireless networks," In Proceedings of the 2012 National Conference on Communications, pp. 1-5, 2012.

[74] J. Sartthong and S. Sittichivapak, "Backoff algorithm optimization for IEEE802.11 wireless local area networks," In Proceedings of the 9th International Conference on Electrical Engineering/Electronics, Computer, Telecommunications and Information Technology, pp. 1-4, 2012.

[75] J. Sartthong, S. Sittichivapak, A. Kaewpukdee and I. Boonpikum, "Binary Exponential Increment Half Decrement backoff algorithm for IEEE802.11 wireless LANs," In Proceedings of the 10th International Conference on Electrical Engineering/Electronics, Computer, Telecommunications and Information Technology, pp. 1-6, 2013.

[76] R. T. Chekka, T. Miao and K. H. Kim, "Implementation of adaptive binary exponential backoff (ABEB) algorithm with dynamical sizing buffer for load-balanced RPL," In Proceedings if the sixth International Conference on Ubiquitous and Future Networks, pp. 562-564, 2014.

[77] Q. Liu and A. Czylwik, "Study on adaptive priority-based servicedifferentiation scheme for IEEE 802.15.4 Wireless Sensor Networks," In Proceedings of the 2014 IEEE Symposium on Computers and Communications, pp. 1-6, 2014.

[78] E. D. N. Ndih and S. Cherkaoui, "Adaptive 802.15.4 backoff procedure to survive coexistence with 802.11 in extreme conditions," In Proceedings of the 13th IEEE Annual Consumer Communications Networking Conference, pp. 556-561, 2016.

[79] Y. Huang, Y. Wang, R. Zhu, X. Chen and Q. Meng, "Synchronized contention windows-based backoff algorithm in IEEE 802.11 wireless networks," In Proceedings of the International Conference on Computer, Information and Telecommunication Systems, pp. 1-5, 2016.

[80] M. Guennoun, M. Khanafer and H. T. Mouftah, "Modeling of Variable Clear Channel Assessment MAC Protocol for Wireless Sensor Networks," Elsevier Computer Communications, Volume 59, March 2015.

\section{APPENDIX A}

In Section IV, Equations (27) and (28) are used to formulate the reliability $(R)$ for a WSN operating the Adaptive Backoff Algorithm (ABA). In this appendix we show the detailed derivations of the probabilities of having $m+1$ backoffs and/or $n+1$ transmission retries [60].

Based on Equation (27), we can write the following set of equations:

$z\left(\pi_{s}+\pi_{B_{1}}+\cdots+\pi_{B_{m+1}}+\pi_{C}\right)=\pi_{s}$

$y\left(\pi_{s}+\pi_{B_{1}}+\cdots+\pi_{B_{m+1}}+\pi_{C}\right)=\pi_{C}$

$x \pi_{S}+x \pi_{B_{m+1}}+x \pi_{C}=\pi_{B_{1}}$

$x \pi_{B_{1}}=\pi_{B_{2}}$

$x \pi_{B_{m}}=\pi_{B_{m+1}}$

The summation $\pi_{S}+\pi_{B_{1}}+\cdots+\pi_{B_{m+1}}+\pi_{C}$ is equal to 1 because it includes all the states that a node can encounter while attempting to send a packet. Therefore, Equations (A.1) and (A.2) reduce to:

$z=\pi_{s}$ 
$y=\pi_{C}$

Therefore, Equation (A.3) can now be re-written as follows:

$x z+x \pi_{B_{m+1}}+x y=\pi_{B_{1}}$

From Equations (A.4) and (A.5), and by clearly examining Equation (27), we can directly see that $\pi_{B_{m+1}}$ can be expressed in terms of $\pi_{B_{1}}$ as follows:

$\pi_{B_{m+1}}=x^{m} \pi_{B_{1}}$

By solving both Equations (A.8) and (A.9) for $\pi_{B_{m+1}}$, we end up with the following expression:

$\pi_{B_{m+1}}=\frac{x^{m+1}(z+y)}{(1-x)^{m+1}}$

Based on Equation (28), we can write the following set of equations:

$z\left(\pi_{s}+\pi_{C_{1}}+\cdots+\pi_{C_{n+1}}+\pi_{B}\right)=\pi_{s}$

$x\left(\pi_{s}+\pi_{C_{1}}+\cdots+\pi_{C_{n+1}}+\pi_{B}\right)=\pi_{B}$

$y \pi_{S}+y \pi_{C_{n+1}}+\pi_{C_{1}} \pi_{B}=\pi_{C_{1}}$

$y \pi_{C_{1}}+\pi_{C_{2}} \pi_{B}=\pi_{C_{n+1}}$

$y \pi_{C_{n}}+\pi_{C_{n+1}} \pi_{B}=\pi_{C_{n+1}}$

The summation $\pi_{s}+\pi_{C_{1}}+\cdots+\pi_{C_{n+1}}+\pi_{B}$ is equal to 1 because it includes all the states that a node can encounter while attempting to send a packet. Therefore, Equation (A.11) reduces to (A.6) while Equation (A.12) reduces to:

$$
x=\pi_{B}
$$

Therefore, Equations (A.13)-(A.15) can now be re-written as follows:

$y z+y \pi_{C_{n+1}}+x \pi_{C_{1}}=\pi_{C_{1}}$

$y \pi_{C_{1}}+x \pi_{C_{2}}=\pi_{C_{n+1}}$

$y \pi_{C_{n}}+x \pi_{C_{n+1}}=\pi_{C_{n+1}}$

From Equations (A.18) and (A.19), and by clearly examining Equation (28), we can directly see that $\pi_{C_{n+1}}$ can be expressed in terms of $\pi_{C_{1} \text {. }}$ as follows:

$\pi_{C_{n+1}}=\left(\frac{y}{1-x}\right)^{n} \pi_{C_{1}}$

By solving both Equations (A.17) and (A.20) for $\pi_{C_{c+1}}$, we end up with the following expression:

$\pi_{C_{n+1}}=\frac{z\left(\frac{y}{1-x}\right)^{n+1}}{1-\left(\frac{y}{1-x}\right)^{n+1}}$

Finally, by knowing Equations (A.10) and (A.21), Equation (26) from Section IV, and by noticing that $z=1-x-y$, we can formulate $R$ as follows:

$R=\frac{1}{1+\frac{(1-x) x^{m+1}}{\left(1-x^{m+1}\right)(1-x-y)}+\frac{y^{n+1}}{(1-x)^{n+1}-y^{n+1}}}$ 